\title{
Constraint Modeling for Curriculum Planning and Validation
}

\author{
Matteo Baldoni ${ }^{1}$, Cristina Baroglio ${ }^{1}$, Ingo Brunkhorst ${ }^{2}$, \\ Nicola Henze ${ }^{2}$, Elisa Marengo ${ }^{1}$, Viviana Patti ${ }^{1}$ \\ ${ }^{1}$ Dipartimento di Informatica - Università degli Studi di Torino \\ \{baldoni, baroglio, emarengo, patti\}@di.unito.it \\ ${ }^{2}$ L3S Research Center, University of Hannover, Germany \\ $\{$ brunkhorst, henze\}@13s.de
}

December 10, 2008

\begin{abstract}
Curricula authoring is a complex process, involving different actors and different kinds of knowledge. Learners aim at acquiring expertise about some topic of their own interest, and need to perceive that the curriculum they attend will lead them towards their goal; when this does not happen, they become demotivated. Learners are all different, not only in their aims but also in their background knowledge and skills; curricula must carefully be tailored to the learner's individual traits: when this does not happen, curricula are not effective from a pedagogical perspective. On the other hand, it is not possible to leave learners alone in the design of a curriculum because this activity involves both knowledge about the topics to teach, and knowledge about teaching itself. It is one of the tasks of the school to support curricula authoring so to guarantee the correctness of the result w.r.t. the teaching goals and to pedagogical strategies. In this article we face the problem of authoring personalized curricula and propose a modular, layered architecture that accounts for the representation of learning resources, of the domain model, of the learner, and of pedagogical constraints, with the aim of supporting different validation tasks. The representation combines a Semantic Web approach to annotation with a declarative representation in linear temporal logic. The validation layer of the proposed architecture includes different kinds of inter-conceptual, postconstruction verifications, all of which can be realized by means of model checking techniques. The article also reports about a prototype implementation based on the Personal Reader for education, a framework that supplies to its users personalization functionalities implemented in a service-oriented fashion.
\end{abstract}

\section{Introduction}

The birth of the Semantic Web $[18,17]$ brought along standard models, languages and tools for representing and dealing with machine-interpretable semantic descriptions of Web resources. The introduction of machine-processable semantics widens the range of the applicable reasoning techniques, giving a strong new impulse to research on personalization. By exploring various representation facets and reasoning techniques it becomes, in fact, feasible to select and deliver contents in a way that is tailored on the specific user.

An interesting research issue for studies on personalization, that requires the development of original solutions, is curriculum sequencing [61, 39, 25,21, 13], where the term curriculum denotes an integrated course of academic studies. Well-designed curricula must both be attractive for students (because they are appealing and challenging) and, at the same time, respect the pedagogical dictates. Usually, however, there is a gap between the learners and the educational offer of schools [42], which is pedagogically sound but not tailored upon the learners' skill and 
desires, which vary from person to person depending on their previous studies and individual abilities $[56,46]$. On the one hand, the learner needs to perceive that his/her studies will allow him/her to achieve the desired competencies, otherwise he/she will be demotivated. For this reason it is interesting to develop tools for designing personalized curricula, targeted at the acquisition of specific learner's goals. On the other hand, one of the school's functions is to guarantee that proper pedagogical strategies are applied in order to make the acquisition of new expertise as smooth and easy as possible [42]. The validation of curricula is a difficult and time-consuming task, for this reason it is useful to have validation tools that automatically check the learners' curricula against the school pedagogical constraints. The possibility to personalize and to automatically validate curricula reduces the gap between the learner's desires and school-designed curricula.

The automatic support to both these task becomes more important, and more difficult, when one considers open scenarios, where the set of learning resources is unrestricted and unknown in advance. Let us, for instance, consider the Bologna Process [29], promoted by the EU. The Bologna process objective is to foster the mobility of students. The final aim is to give students the possibility of attending integrated curricula, whose courses are scattered among different European institutions. The advantage is that, by exploiting this possibility, students will also be enabled to acquire competences which can only be achieved by composing some complementary knowledges that are supplied only by different academic institutes. So, for instance, an Italian student might needs to move to the University of Hannover for a period, because part of the expertise he/she would like to acquire can only be achieved by attending some courses there. In general, personalization is necessary because students, that are involved in the mobility program, are interested in combining courses so to acquire some desired expertise (the student's learning goal), they come from different schools and have different backgrounds and skills. Their studies will be effective only if all these ingredients are taken into account.

In order to overcome the gap between the learner's desires and the school's pedagogical requirements, and so to meet objectives like those of the Bologna process, it is necessary to develop tools for supporting learners and schools in practice and, in particular, the means for designing curricula (either offered or desired) that suit learners individually, verifying that they are pedagogically sound. To this purpose Semantic Web comes in handy because it supplies the means for describing courses (more in general, learning resources) by means of meta-data [24], i.e. machine-interpretable semantic annotations. In particular, Semantic Web offers standard models and languages for sharing knowledge $[63,62,6,58,47]$. The use of semantic annotations is even more important considering that learning resources are heterogeneous in different ways and have different sources. For instance they can be supplied as different media (textual documents as well as animations), they can have different granularity (single lessons as well as courses) [25], and they can be gathered from different repositories. The representations based on Semantic Web languages allow the application of different kinds of automatic reasoning techniques, mostly deriving from studies on Artificial Intelligence: from ontological reasoning [6] to techniques that lay at the logic and proof layers of the Semantic Web tower [5], and that exploit rule-based representations $[48,57]$.

In this article we present the results of a work (carried on in the context of the REWERSE network of excellence [52]) that concerns the representation of learning resources, of the domain model, and of pedagogical constraints so as to allow the design of personalized curricula, that are sound w.r.t. the pedagogical constraints given by a school. In particular, along the lines of $[46,45]$, we structure curricula authoring in a set of representational and activity layers. The lowest layer, the domain model, captures information about the domain to be taught but without taking into account any pedagogical information. For instance, it could be an ontology (or a taxonomy) describing the topic. On top of this, the curricula model (goal and constraint model in $[46,45])$ specifies the knowledge to be delivered, from the point of view of the school, and the pedagogical constraints - specified by some instructional designer -, that rule the order in which concepts are to be taught and that define some other relations among the acquisition of concepts. In order to represent constraint models we propose the language DCML (Declarative Curricula Model Language), a graphical language with a grounding in linear temporal logic [28], that allows the representation of many kinds of constraints among competencies. The learner 
model contains the knowledge of the learner and his/her learning goal, expressed in terms of the concepts in the domain model. The resource model specifies, for each learning resource (for instance, for each course) its preconditions, i.e. the knowledge that a learner should have for using the resource with profit, and its effects, i.e. the knowledge that is supplied by the resource. The curriculum (course model in $[46,45]$ ) is described in terms of the learning resources contained in the resource model. We use UML activity diagrams to represent curricula. The validation model defines the pedagogical strategies that are to be enforced. In particular, we tackle: the verification of the goal-achievement property, i.e. that a curriculum allows either the achievement of the learner's goal or the achievement of the school learning goal; the competence gap problem, i.e. that at every step a learner attending a curriculum will always have the knowledge that is necessary for attending the next course; the respect of the constraints in the constraint model. Moreover, on this same representation it is possible to perform also planning tasks, thought as a means to help learners in the construction of personalized curricula. A prototype implementation is described in Section 6. This implementation relies on the Personal Reader (PR) framework [38], a service-oriented framework that supplies to the final user web services for dealing with semantic information. The Personal Reader framework also allows for the combination of services into new personalization functionalities by exploiting the concept of syndication.

The article is organized as follows. Section 2 gives an overview of our proposal by presenting an architecture for curricula authoring and by introducing our implementations of the various layers. The subsequent sections present in details such realizations. In particular, Section 3 presents a constraint-based representation of curricula models, and the DCML language, including both the graphical and the LTL characterizations. Further constraints that can be used to enrich curricula models are reported in Section 7. Section 4 shows how curricula are represented by means of UML activity diagrams, reporting some examples. Section 5 describes in details the validation layer and the implementation of the validation tasks, with the help of some Promela code. Section 6 describes, instead, the integration steps performed to implement part of the curricula authoring architecture in the Personal Reader. A comparison with relevant works in the literature and some conclusive remarks end the article.

\section{Curricula authoring}

Curricula authoring is a complex process that involves different actors and different kinds of knowledge. Returning to the Italian student, who needs to attend some courses in Germany, nowadays the student would be guided by an Italian mentor in the definition of a curriculum, which combines the student's interests, the educational goals of the Italian school, the courses offered by the same institute, those offered by the German hosting institute, and also the guidelines and constraints posed by the latter. Quite a complex and time-consuming task [19]. The work that we propose aims at making the execution of this task automatic. Along the lines of [46, 45], in order to simplify curricula authoring we structure this process into a layered architecture (see Figure 1). The adoption of a structured view has the advantage of modularizing the process, making the dependencies between different kinds of knowledge and of tasks clear and explicit. In this way, the upgrades (modifications) become simpler, because they affect single modules, and the effects of such modifications on the overall process can be evaluated more easily.

\section{Domain Model}

The base level is the domain model. This layer contains the knowledge about the domain itself expressed as a set of concepts and their relations, independently from any pedagogical concern. Concepts can, for instance, represent the various topics and their organization in sub-topics. Concepts can easily be given a semantic representation by using the tools and the languages supplied by the Semantic Web community. The domain model used in this work (see Section 6.1), is a vocabulary of RDF terms, that have been extracted automatically by means of the Lixto tool [16], applied to the descriptions of the courses held at the University of Hannover, that are 


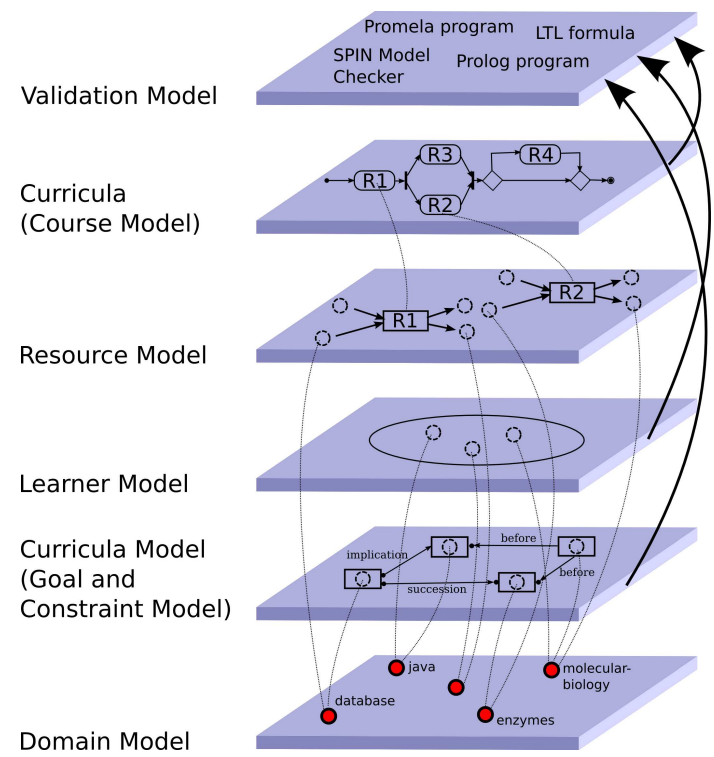

Figure 1: The layered architecture.

supplied by HIS-LSF (http://www.his.de). So far, Lixto returns a flat RDF vocabulary. In the future, it might be interesting to try to derive an ontology from the corpus by structuring the concepts [49, 4], e.g. by using Levenshtein distances and a thesaurus, like wordnet. Focussing on a flat vocabulary is not restrictive because, due to the adoption of a modular (layered) architecture, one can think to integrate ontological reasoning in the domain model layer and, as a consequence, to have it included also in the higher levels.

In the literature concerning professional curricula and e-learning, concepts are referred to either as competency (plural "competencies") or as competence (plural "competences"), to denote respectively "any form of knowledge, skill, attitude, ability or learning objective that can be described in a context of learning, education or training" and the "effective performance within a domain at some level of proficiency" [26]. Our domain model contains competencies. Different courses can supply/require the same competency at different proficiency levels. For the sake of readability, in this article we do not use RDF to represent competences; instead, we use a more intuitive notation of the kind (database, beginner), where the first element denotes a concept while second a proficiency level.

\section{Curricula Model (Goal and Constraint Model)}

The subsequent layer (Figure 1) is the curricula model, which contains pedagogical constraints defined over the domain model. The curricula model includes a set of constraints on competences (i.e. concepts plus a proficiency level). We partition constraints into a set of requirements, a set of goals, and a set of relational constraints. The requirements specify the expected competences that a learner should have before starting the learning process. The goals specify the knowledge that is to be delivered. The relational constraints structure the teaching activity of the various concepts in the domain model, by imposing orderings or presence. In particular, we have identified three kinds of relational constraint (and their negations): before, implication, and succession (Figure 4). The first kind of constraint expresses the fact that in order to acquire a competence, another competence must be owned before. Implication constraints express the fact that if at some point a competence is acquired, also some other competence must already be owned by the learner or it must be learnt in the future. Finally, succession constraints express the fact that when a competence is acquired, then another is to be be acquired in the future. The expressiveness of the proposed representation makes curricula models not only sets of precedence constraints, as done 
in previous work (e.g. $[46,45]$ ), but it makes them rich schemas - see Figure 2 -, each of which defines a whole class of curricula.

Curricula models are represented by means of the Declarative Curricula Modeling Language (DCML for short, see Section 3). DCML is a graphical language (Figure 2 shows an example), inspired by DecSerFlow, the Declarative Service Flow Language to specify, enact, and monitor web service flows by van der Aalst and Pesic [60]. DCML, as well as DecSerFlow, is grounded in Linear Temporal Logic [28] and allows a curricula model to be described in an easy way maintaining at the same time a rigorous and unambiguous meaning given by the logic representation. The use of a logic with a clear semantics allows the separation of the representation of constraints from the choice of an algorithm to perform validation tasks. As an effect the curricula model layer and the validation layer result more independent.

\section{Learner Model}

The learner model represents the knowledge of a learner, which evolves during the learning process. Intuitively, by attending a course the learner will gain the knowledge taught in that course. Before starting the learning process, this representation is set to the initial expertise of the learner. So, the learner model contains a representation of the mental state of the learner. The expectation of the learner is that its mental state will evolve so to include some desired expertise, i.e. so to reach a specific learning goal. Also the learning goal is included in the learner model. The learner's knowledge and learning goals are expressed as sets of competences.

\section{Resource Model}

The resource model describes courses, i.e. our learning resources, in terms taken from the domain model. Specifically, each course is described in an action-based fashion [10, 14, 13, 9, 12], by its preconditions and effects. Both preconditions and effects are competences. As such, each of them is a pair that combines a competency (any form of knowledge, skill, attitude, ability or learning objective that can be described in a context of learning, education or training) with a proficiency level. This representation is general because, although in this paper we deal with courses, it expresses an information that lays at the knowledge level and, for this reason, it can be used to tackle any kind of learning resource. As an example of resource representation, let us consider a course with name Biology_II, which requires beginner's knowledge about protein_structures and some deeper (intermediate level) knowledge about em cell_structure. The course supplies advanced knowledge about enzymes and about molecular_biology, and intermediate knowledge about immune_systems. It will be represented as follows:

resource_name: Biology_II

preconditions: (cell_structure, intermediate), (protein_structures, beginner)

effects: (enzymes, advanced), (immune_systems, intermediate), (molecular_biology, advanced)

Preconditions are evaluated against the representation of the learner's mental state, that is contained in the learner model, while effects modify this representation, causing an evolution of the learner's knowledge.

In an open environment, it is likely that resources belonging to different schools are annotated by using different domain models, which are, therefore, to be combined in some way in order to obtain a uniform representation that allows the application of reasoning techniques [36]. Supposing that domain models are given as domain ontologies, it becomes possible to apply techniques for ontology merging, alignment and integration, e.g. [30]. Indeed, these are hot research topics, that we do not tackle in this work. We make the hypothesis that this alignment already occurred and that all resources are represented in terms of a common ontology.

\section{Curriculum (Course Model)}

Curricula (see Figure 5 for an example) are represented by means of UML activity diagrams [2], which compose resources, whose representation is supplied by the previous layer. In the simplest 
case, a curriculum can be seen as a sequence of actions that causes transitions from the initial set of competences (possibly empty) of a user up to a final state that will contain also the acquired competences. We assume that concepts can only be added to states and competence level can only grow by executing the actions of attending courses (or more in general reading a learning material). The intuition behind this assumption is that new courses do not erase the concepts acquired previously, thus knowledge grows incrementally. Generally speaking, a curriculum may contain one or several learning pathways to be attended, in alternative or as obligations.

The use of UML activity diagrams seems very natural for representing curricula, and they are used also in other works to this purpose, e.g. [46]. As a difference, we found two principles very useful when representing a curriculum (Section 4): to carefully distinguish courses with distinct duration (in time); to carefully distinguish mandatory courses and optional courses. Modelling a curriculum with these two principles in mind introduces (i) a decomposition level and (ii) partitions among courses, being these courses from mandatory or from optional partitions.

\section{Validation Model}

Finally, we have the validation model. According to the CAVIAr model [46, 45], pedagogical validation can be intra-conceptual (ensuring that each concept is taught in a uniformed manner), inter-conceptual (concerning how the curriculum proceeds from one concept to another), or it can be done w.r.t. pedagogical rules which specify the types of resource that are involved (e.g. resources of type "lecture"). With reference to this classification, the kinds of validation that we perform are inter-conceptual. In particular, for what concerns curricula authoring three are the main checks to perform [21]: (a) verify that the curriculum brings to the acquisition of the desired knowledge; (b) verify that at every point the learner already has the knowledge, that is necessary for understanding the next taught concept; (c) verify that the curriculum respects the goal and constraint model. Task (a) allows checking whether a curriculum brings to the achievement of the declared user's learning goal. In this case, the verification process uses the learner model (that, in turn, uses the domain model) and the course model (that, in turn, uses the resource model, which uses the domain model). Task (b) consists in checking that the curriculum shows no competence gaps [26], i.e. the knowledge that is necessary to fully understand a course is introduced or available before it is attended. In this case, the verification process uses the course model, which uses the resource model, which, in turn, uses the domain model. Task (c) verifies the compliance of a given curriculum against the guidelines (pedagogical strategies) given by the institution [21], a tedious task that is currently performed manually. It uses the course model, and the curricula model, and verifies that the curriculum represented by the course model satisfies all the constraints imposed by the other model.

To perform all these tasks, we use model checking techniques [23] (Section 5). By means of a model checker, it is possible to generate and analyze all the possible states of a model (represented as an automaton) exhaustively, to verify that all execution paths satisfy a certain property, usually expressed by a temporal logic, such as LTL. In our case, the model is a curriculum (i.e. it is the course model), suitably translated into an automaton. The LTL formula is, instead, obtained in different ways depending on the task to accomplish. For Task (a), it is obtained by translating the learning goal (which is part of the learner model) into a set of assertions that are to be satisfied by the final states of the automaton. For Task (b), the resource model is translated into a set of assertions that are to be satisfied by the states in the automaton. For Task (c), it is the goal and constraint model, which is translated into a set of LTL formulas that the automaton must satisfy.

When a model checker finds that a model does not satisfy some LTL formula (or assertion), it produces a counterexample that shows the violation. Even though it is possible to argue that model checking techniques are not efficient because they analyze the whole space of possible executions, the fact of having counterexamples is extremely important because it supplies an explanation of the failure, that can be supplied as feedback for the user. It is well-known that the user will not be confident in the answer unless he/she can trust the reasons why the answer was produced. As a model checker, in this work we have used SPIN, by G. J. Holzmann [40], which is the most representative tool of this kind. 


\section{Implementation and planning}

All the kinds of validation mentioned above are post-construction course validations [50, 54], that can be used to produce personalization functionalities. To this aim, we have developed a prototype implementation (Section 6), based on the PR (Personal Reader) framework [38] - see Figure 7 for an overview -. The PR relies on a service-oriented architecture enabling personalization, via the use of Personalization Services, that communicate solely based on RDF documents. Each service offers a different personalization functionality, e.g. recommendations tailored to the needs of specific users, pointers to related (or interesting or more detailed/general) information, and so on. Besides the verification functionalities, the PR offers a service for automatically composing a curriculum, based on the learner model and on the semantic annotation supplied by the resource model, that exploits automatic planning techniques [55]. In fact, by interpreting resources as actions, a curriculum can, in turn, be interpreted as a plan, whose execution causes transitions from a state to another, until some final state is reached. A transition between two states is due to the application of an action, that corresponds to attending a course. To apply an action, its preconditions must hold in the state to which it is applied, and its application consists in an update of the state. We assume that facts can only be added to states. The intuition behind this assumption is that the act of attending a course (or, more in general, of using a learning resource) will not erase from the learner's memory the concepts acquired so far. The service that we have developed, that is described in details in Section 6.3, is specifically aimed at building linear curricula, i.e. curricula which consist of a sequence of courses only. Such curricula can be tailored to a specific user by starting from an initial state that contains that user's knowledge. This perspective is taken in several works that face the so called curriculum sequencing problem $[39,21,61]$, that is the problem of generating a personalized learning path for each student, by dynamically selecting at any moment a resource that, in the context of other available resources, brings the student closer to the learning goal.

\section{Curricula Models and DCML}

In order to present curricula models, we propose a constraint-based representation. The instructional designer can express various kinds of constraints by means of a simple graphical language, that we call DCML (Declarative Curricula Model Language). DCML constraints can be automatically turned into formulas in a temporal logic (LTL, linear temporal logic [28]). This logic allows the verification of properties of interest for all the possible executions of a model, which in our case corresponds to a curriculum. LTL includes temporal operators such as next-time $(\bigcirc \varphi$, the formula $\varphi$ holds in the immediately following state of the run), eventually $(\nabla \varphi, \varphi$ is guaranteed to eventually become true), always ( $\square \varphi$, the formula $\varphi$ remains invariably true throughout a run), until ( $\alpha \mathrm{U} \beta$, the formula $\alpha$ remains true until $\beta$ ), see also [40, Chapter 6]. The adoption of a graphical language with a logical grounding allows designers, who cannot be expected to feel comfortable with the logical notation, to take anyway advantage of automatic verification tools for accomplishing the tasks foreseen by the validation model. On the other hand, relying on a logic with a rigorous semantics allows a separation of concerns between the constraint model and the choice of an algorithm (tool) for performing the validation tasks. Constraints have a declarative semantics which is independent from the implementation.

Constraint-based representations are very compact because they require to express only those necessary conditions that characterize a correct curriculum, avoiding overspecification. To make an example, by means of constraints we can request that a certain knowledge is acquired before some other knowledge, without expressing what else is to be done in between. This is an advantage w.r.t. other approaches, like the procedural approach (e.g. [13]), having a prescriptive nature because in the latter it is necessary to express exhaustively all the pathways that are correct. If, on the one hand, it makes sense that some topics have a specific ordering, it is also intuitive that this does not happen for all the involved competences. For example, it makes sense that the competence (database, beginner) (knowledge about databases with proficiency level "beginner") 


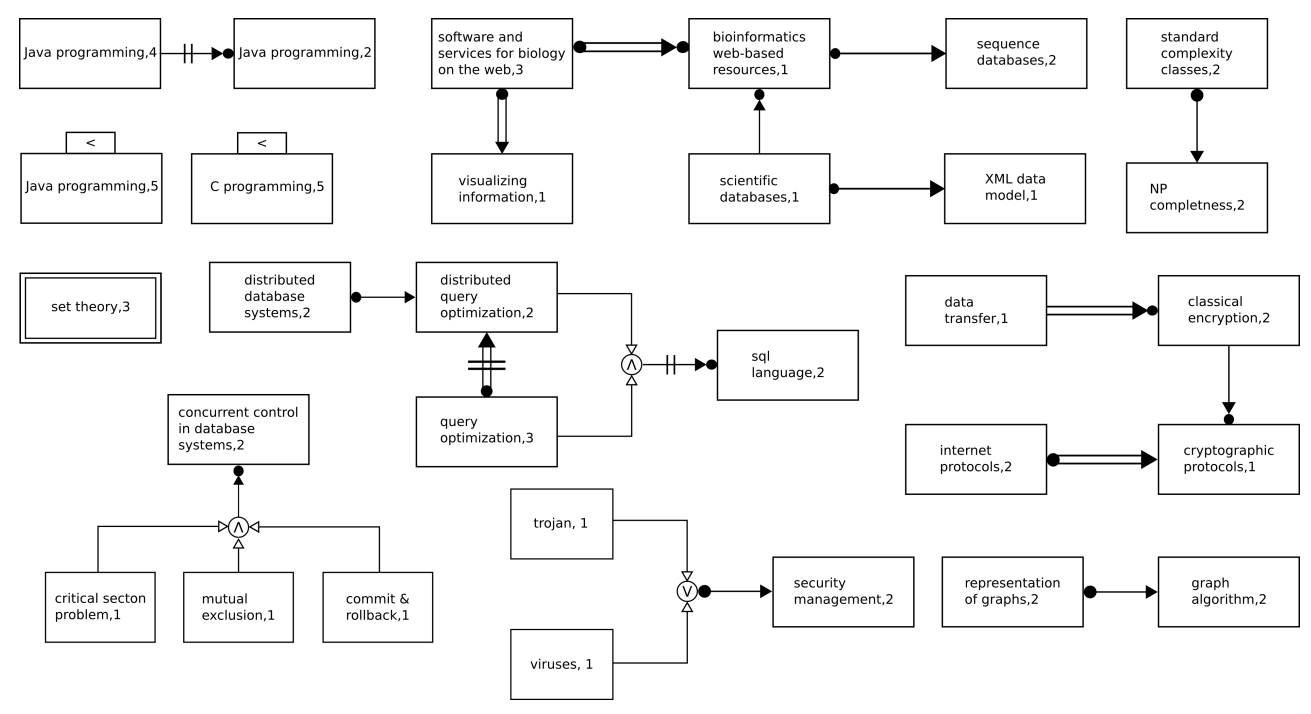

Figure 2: An example of curricula model in DCML.

is to be acquired before (database, advanced) but what about a competence (English, advanced)? Should it be acquired before or after the other two mentioned competences? Intuitively, it is not necessary to impose that (English,advanced) is, for instance, the first competence to acquire because it is not related to the other topics. The problem is that an ordering that is not explicitly mentioned in the model is not legal, so the designer must foresee many variants within the schema. By using constraints, in the previous case, we only need to express that (database, beginner) must be acquired before (database, advanced) and that (English, beginner) is to be acquired sooner or later. For this reason, we can say that the constraints-based approach is more flexible and more suitable in an open environment, due to the fact that in an open environment resources are many, they are various, and they are added and removed dynamically.

As an example, Figure $2^{1}$ shows a curricula model expressed in DCML. Every box contains at least one competence. Boxes/competences are connected by arrows, which represent (mainly) temporal constraints among the times at which they are to be acquired. Altogether the constraints describe a curricula model. Let us now introduce DCML in details.

\subsection{Expressing competences and basic constraints}

DCML is used to define a set of constraints on top of the domain model. Competencies are associated to a representation of the proficiency level at which a competency is owned or to be supplied. So, for instance, we express the fact that a competency database is to be owned at level beginner. To simplify the management of proficiency levels, such levels are represented as numerical values. To this aim, we associate to each competency a variable $k$, having the same name as the competency, which can be assigned natural numbers as values. The value of $k$ denotes the proficiency level; zero means absence of knowledge. Therefore, $k$ encodes a competence, Figure 3. On top of competences, in DCML it is possible to define two basic constraints:

- Goal constraints: A goal constraint - Figure 3, third row - imposes that a certain competency $k$ must be acquired at least at level $l$. It is represented by the LTL formula $\diamond(k \geq l)$. Similarly, a course designer can impose that a competency must never appear in a curriculum with a proficiency level higher than $l$. This is possible by means of the "always less than level" constraint, shown in Figure 3 fourth row. The LTL formula $\square(k<l)$ expresses this

\footnotetext{
${ }^{1}$ Notice that double arrows are defined in Section 7.
} 


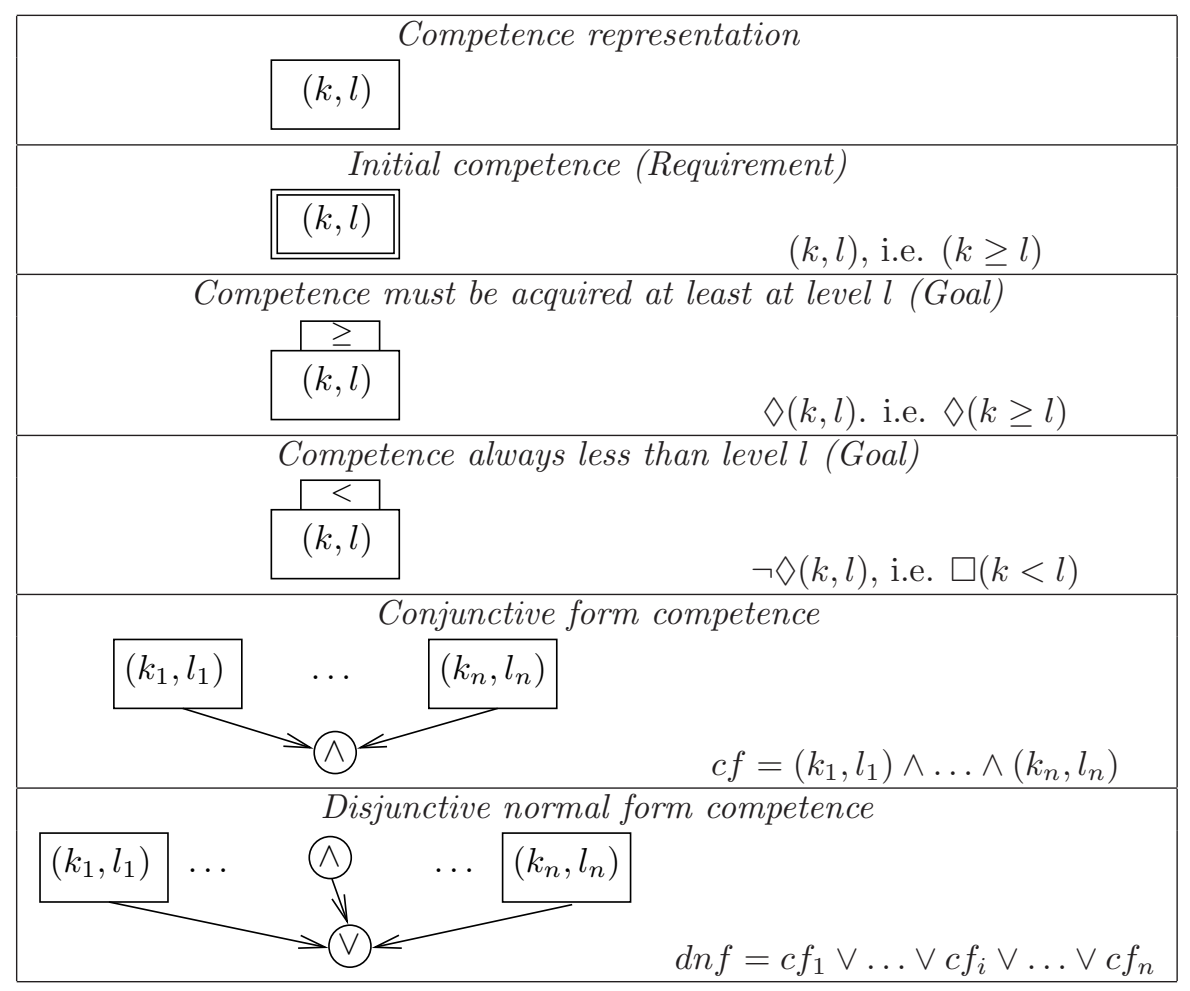

Figure 3: DCML representation of competences, conjunctions and disjunctions of competences.

fact (it is the negation of the previous one). As a special case, when the level $l$ is one $(\square(k<1))$, the competency $k$ must never appear in a curriculum.

- Requirements: A requirement, represented by a double box - see Figure 3, second row specifies that before starting the learning process, the learner must own the competence $k$ at least at level $l$. This is represented by the logic formula $(k \geq l)$.

In DCML it is possible to represent Disjunctive Normal Form (DNF) formulas as conjunctions and disjunctions of competences ${ }^{2}$. Graphically, a conjunction of basic constraints is represented by a circle with a " $\wedge$ " symbol inside, having as many incoming arrows as conjuncts. A disjunction, instead, can compose both basic constraints and conjuncts of constraints. Graphically - Figure 3, last two rows - it is represented by a circle with a " $\vee$ " symbol inside, the disjuncts are connected to it by means of arrows.

Let $k$ be a competence, we denote by $(k, l)$ the constraint $k \geq l$ and by $\neg(k, l)$ the constraint $k<l$. A conjunctive competence formula $c f$ is a conjunction of atomic competence constraints $c f=\left(k_{1}, l_{1}\right) \wedge \cdots \wedge\left(k_{n}, l_{n}\right)$. A conjunction can also be interpreted as the set of constraints $c f=\left\{\left(k_{1}, l_{1}\right), \ldots,\left(k_{n}, l_{n}\right)\right\}$. To help the expression of LTL formulas we introduce the functions:

- $\operatorname{negation}(c f)=\bigwedge_{\left(k_{i}, l_{i}\right) \in c f} \neg\left(k_{i}, l_{i}\right)$;

- existence $(c f)=\bigwedge_{\left(k_{i}, l_{i}\right) \in c f} \diamond\left(k_{i}, l_{i}\right)$;

- absence $(c f)=\bigwedge_{\left(k_{i}, l_{i}\right) \in c f} \square \neg\left(k_{i}, l_{i}\right)$.

A disjunctive normal competence formula $d n f$ is a disjunction of conjunctive competence formulas, $d n f=c f_{1} \vee \cdots \vee c f_{n}$. Again, we also denote a disjunctive normal competence formula as a set of conjunctive competence formulas $d n f=\left\{c f_{1}, \ldots, c f_{n}\right\}$. Therefore, a disjunctive normal competence formula is a set of sets of atomic competences.

\footnotetext{
${ }^{2} \mathrm{~A}$ DNF formula is an expression having the form $\left(a_{1,1} \wedge a_{1,2} \wedge \ldots\right) \vee\left(a_{2,1} \wedge a_{2,2} \wedge \ldots\right) \vee \ldots$
} 


\subsection{Constraints among competences}

Besides the representation of competences and of constraints on competences, DCML allows the representation of relations among competences. These relations are represented as different kinds of arrows. It is also possible to represent "negative relations" by using two vertical lines to break the arrow that represents the constraint.

There are three main kinds of relation:

- before: this relation expresses the fact that in order to acquire a competence, another competence is to be owned in advance;

- implication: this relation means that if at some point a competence is acquired, also some other competence must already be owned by the learner or it must be learnt in the future;

- succession: means that when a competence is acquired, then another is to be be acquired in the future.

\section{Before}

Arrows ending with a little-ball, Figure 4, express the before temporal constraint between two competences. " $\left(k_{1}, l_{1}\right)$ before $\left(k_{2}, l_{2}\right)$ " requires that $\left(k_{1}, l_{1}\right)$ holds before $\left(k_{2}, l_{2}\right)$. This constraint can be used to express that to understand some topic (e.g., $k_{2}$ at least at the level $l_{2}$ ), some proficiency of another is required as precondition (in the example, $k_{1}$ at least at the level $l_{1}$ ). It is important to underline that if the antecedent never becomes true, also the consequent must be invariably false; this is expressed by the LTL formula $\neg\left(k_{2}, l_{2}\right) \cup\left(k_{1}, l_{1}\right)$. More generally, in presence of DNF formulas as antecedent and consequence of a "before" relation, we have the following definition for " $d n f_{1}$ before $d n f_{2}$ ":

$$
\bigvee_{c f_{i} \in d n f_{1}, c f_{j} \in d n f_{2}} \text { negation }\left(c f_{j}\right) \cup c f_{i}
$$

Example 3.1 With reference to Figure 2 - bottom-left side of the picture, the competences (critical_section_pro-blem, 1), (mutual_exclusion, 1) and (commitErollback, 1) are necessary for the user to understand the competence (concurrent_control_in_db_systems, 2). Hence, the curricula model contains a constraint: (critical_section_problem, 1) $\wedge$ (mutual_exclusion, 1) $\wedge($ commitGrollback, 1) before (concurrent_control_in_db_systems, 2)

A constraint of kind " $\left(k_{1}, l_{1}\right)$ not before $\left(k_{2}, l_{2}\right)$ " specifies that $k_{1}$ cannot be acquired up to level $l_{1}$ before or in the same state when $\left(k_{2}, l_{2}\right)$ is acquired. The corresponding LTL formula is $\neg\left(k_{1}, l_{1}\right) \cup\left(\left(k_{2}, l_{2}\right) \wedge \neg\left(k_{1}, l_{1}\right)\right)$. Notice that this is not obtained by simply negating the before relation but it is weaker; the negation of before would impose the acquisition of the concepts specified as consequents (in fact, the formula would contain a strong until instead of a weak until), the not before does not. More generally, in presence of DNF formulas, " $d n f_{1}$ not before $d n f_{2}$ " is:

$$
\bigvee_{c f_{i} \in d n f_{1}, c f_{j} \in d n f_{2}} \text { negation }\left(c f_{i}\right) \cup\left(c f_{j} \wedge \text { negation }\left(c f_{i}\right)\right)
$$

Example 3.2 As an example, the constraint (distributed_query_optimization, 2) $\wedge$ (query_optimization, 3) not before (sql_language, 2) represents the fact that competences (distributed_query_optimization, 2) and (query_optimization, 3) cannot be acquired before or in the same state in which the competence (sql_language, 2) is acquired. The reason could be that distributed_query_optimization and query_optimization are more difficult topics and learners need time to get acquainted with them.

Example 3.3 Another interesting way to use this constraint is described by the following example: (Java_pro-gramming, 4) not before (Java_programming, 2). In this way we express that the competency Java_programming is too important (in a certain curricula model) and from a pedagogical point of view, it cannot be acquired immediately at advanced level. Instead, the student has to acquire it in two subsequent steps: intermediate (proficiency level 2) and then advanced (proficiency level 4 in the example). 


Before: $\left(k_{1}, l_{1}\right)$ before $\left(k_{2}, l_{2}\right)$
$\neg\left(k_{2}, l_{2}\right) \cup\left(k_{1}, l_{1}\right)$
Competence $\left(k_{1}, l_{1}\right)$ must be acquired before or at the
same time of competence $\left(k_{2}, l_{2}\right)$

Figure 4: DCML representation of basic constraints among competences. Arrows can connect not only competences but also conjunctions and disjunctions of competences, see Figure 2. 


\section{Implication}

Before relation represent temporal constraint between competences. The implication relation, for example " $\left(k_{1}, l_{1}\right)$ implies $\left(k_{2}, l_{2}\right)$ ", denoted by arrows starting with a little-ball (see Figure 4 ), specifies, instead, that if a competency $k_{1}$ holds at least at the level $l_{1}$, some other competency $k_{2}$ must be acquired, sooner or later, at least at the level $l_{2}$. The main characteristic of the implication, is that the acquisition of the consequent is imposed by the truth value of the antecedent, but, in case this one is true, it does not specify when the consequent must be achieved (it could be before, after or in the same state of the antecedent). This is expressed by the LTL formula $\diamond\left(k_{1}, l_{1}\right) \supset \diamond\left(k_{2}, l_{2}\right)$. More generally, in presence of DNF formulas as antecedent and consequence of a "implication" relation, we have the following definition for " $d n f_{1}$ implies $d n f_{2}$ ":

$$
\bigvee_{c f_{i} \in d n f_{1}, c f_{j} \in d n f_{2}} \operatorname{existence}\left(c f_{i}\right) \supset \text { existence }\left(c f_{j}\right)
$$

To have a better intuition, consider the two algorithms breadth_first_search and depth_first_search. Of course, it is not necessary to know one of them in order to understand the other but with the implication we can impose that if one of them is acquired, also the other one must be owned by the student before the end of the curriculum: (breadth_first_search, l1) implies (depth_first_search, 12).

Example 3.4 Let us consider the constraint, Figure 2, (distributed_database_systems, 2) implies (distributed_query_optimization, 2). This constraint imposes that if the competence (distributed_database_systems, 2) is acquired, also (distributed_query_optimization, 2) must be acquired sooner or later. Generally, the implication constraint is useful to express that when a learner acquires a certain competence, for the sake of completeness, the learner should also have another competence; if he/she does not have it yet he/she will have to acquire it by the end of the curriculum.

" $\left(k_{1}, l_{1}\right)$ not implies $\left(k_{2}, l_{2}\right)$ " expresses that if $\left(k_{1}, l_{1}\right)$ is acquired $k_{2}$ cannot be acquired at level $l_{2}$; as an LTL formula: $\diamond\left(k_{1}, l_{1}\right) \supset \square \neg\left(k_{2}, l_{2}\right)$. Again, we choose to use a weaker formula than the natural negation of the implication relation because the simple negation of formulas, $\diamond\left(k_{1}, l_{1}\right) \wedge \square \neg\left(k_{2}, l_{2}\right)$, would impose the presence of certain concepts $\left(k_{1}\right.$ at least at level $l_{1}$, in the example). More generally, in presence of DNF formulas, " $d n f_{1}$ not implies $d n f_{2}$ " is:

$$
\bigvee_{c f_{i} \in d n f_{1}, c f_{j} \in d n f_{2}} \text { existence }\left(c f_{i}\right) \supset \text { absence }\left(c f_{j}\right)
$$

This kind of constraint is useful to express the fact that two competences A and B, considered as equivalent to one another, should be mutually exclusive. We can capture this by using the pair of constraints: $A$ not implies $B$, and $B$ not implies $A$. This is analogous, though at the level of competences and not at the level of courses, to a kind of constraint proposed in [64], expressing the fact that equivalent courses are not to be used in a same curriculum.

\section{Succession}

The last constraint is succession (arrows starting and ending with a little-ball, Figure 4). " $\left(k_{2}, l_{2}\right)$ succeeds $\left(k_{1}, l_{1}\right)$ " specifies that if $\left(k_{1}, l_{1}\right)$ is acquired, afterwards $\left(k_{2}, l_{2}\right)$ is also achieved; otherwise, the level of $k_{2}$ is not important. This is a difference w.r.t. the before constraint where, when the antecedent is never acquired, the consequent must be invariably false. Indeed, the succession specifies a condition of the kind if $k_{1} \geq l_{1}$ then $k_{2} \geq l_{2}$, while before represents a constraint without any conditional premise. Instead, the fact that the consequent must be acquired after the antecedent is what differentiates implication from succession. Succession constraint is expressed by the LTL formula $\diamond\left(k_{1}, l_{1}\right) \supset\left(\diamond\left(k_{2}, l_{2}\right) \wedge\left(\neg\left(k_{2}, l_{2}\right) \cup\left(k_{1}, l_{1}\right)\right)\right)$. More generally, in presence of DNF formulas as antecedent and consequence of a "succession" relation, we have the following definition for " $d n f_{2}$ succeeds $d n f_{1}$ ":

$$
\bigvee_{c f_{i} \in d n f_{1}, c f_{j} \in d n f_{2}} \operatorname{existence}\left(c f_{i}\right) \supset\left(\text { existence }\left(c f_{j}\right) \wedge \text { "c } f_{i} \text { before } c f_{j} "\right)
$$


This constraint specifies an ordering on the acquisition of two competences. It can be used, for instance, to express a pedagogical constraint stating that after a learner has acquired some theoretical competence about a specific topic, the same learner must also acquire some practical skill about the same topic.

Example 3.5 An example, which is not included in Figure 2 is the following: (Oracle_DBMS, l1) succeeds (DBMS, 12). The constraint specifies that after the acquisition of knowledge about DBMS (at the proficiency level $l 1$ ), also skills about the Oracle DBMS is to be acquired (at the proficiency level $l 2$ ).

" $\left(k_{2}, l_{2}\right)$ not succeeds $\left(k_{1}, l_{1}\right)$ " imposes that a certain competence, $\left(k_{2}, l_{2}\right)$, cannot be acquired after another, $\left(k_{1}, l_{1}\right)$, either it was acquired before, or it will never be acquired. As LTL formula, it is $\diamond\left(k_{1}, l_{1}\right) \supset\left(\square \neg\left(k_{2}, l_{2}\right) \vee\right.$ " $\left(k_{1}, l_{1}\right)$ not before $\left(k_{2}, l_{2}\right)$ "). Similarly to the previous negative constraints, we choose to use a weaker formula than the natural negation of the succession relation because the simple negation of formulas, $\diamond\left(k_{1}, l_{1}\right) \wedge\left(\square \neg\left(k_{2}, l_{2}\right) \vee \neg\right.$ " $\left(k_{1}, l_{1}\right)$ before $\left(k_{2}, l_{2}\right)$ "), would impose the presence of certain concepts $\left(k_{1}\right.$ at least at level $l_{1}$, in the example). More generally, in presence of DNF formulas, " $\operatorname{dnf} f_{2}$ not succeeds $d n f_{1}$ " is:

$$
\bigvee_{c f_{i} \in d n f_{1}, c f_{j} \in d n f_{2}} \operatorname{existence}\left(c f_{i}\right) \supset\left(\text { absence }\left(c f_{j}\right) \vee \text { "c } f_{i} \text { not before } c f_{j} "\right)
$$

\section{Representation of Curricula}

In general, a curriculum may be represented with one or several learning paths to be attended, optionally or as an obligation. We represent curricula by means of UML activity diagrams [2]. The diagram captures essentially the "student personal process" to achieve the final degree. In the simplest case, a curriculum is a sequence of courses, that we represent as activities in the resource model (see Section 2, Resource Model), causing transitions from the initial set of competences (possibly empty) of a learner (included in the learner model) up to a final state that will contain the acquired competences. As mentioned, we assume that, as an effect of attending courses, competences are only added to states and that competence levels can only grow. Along the line of $[13,9,12]$, we interpret courses (activities) as actions, that can be executed given that their preconditions hold. By executing an activity, a set of post-conditions, the effects, will become true (the learner will acquire new competences).

Example 4.1 Let us consider again the example in Section 2:

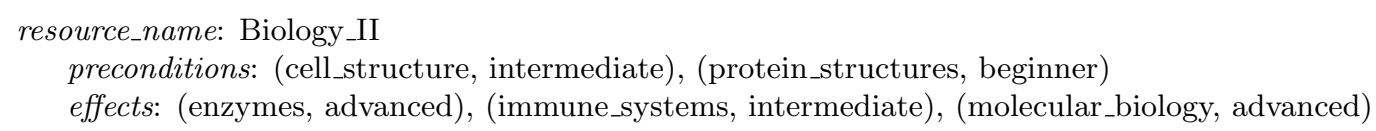

The activity Biology_II is executable at some point of a learning path, if at that point the learner has already acquired the competences (cell_structure, intermediate), (protein_structures, beginner). After the transition, caused by the execution of the activity Biology_II, the learner will be in a state that also includes the competences: (enzymes, advanced), (immune_systems, intermediate), (molecular_biology, advanced).

UML activity diagrams are well suited for representing curricula for many reasons. They may contain activities with pre- and post- conditions, combined in complex paths and possibly aggregated. Activity diagrams are rich enough to represent alternative, intermediate states and conditional paths. Moreover, they allow the distinction of courses with different duration (in time), the distinction of mandatory learning paths and additional (optional) learning paths, and the decomposition of an activity in sub-activities up to the desired granularity.

Figure 5 reports an example. The horizontal partition (swimlanes in UML) allows the distinction of mandatory and additional learning paths. With reference to the example, the curriculum includes three additional pathways (Networks and Programming, Databases, and Bioinformatics), that can alternatively be used to complement the mandatory pathway. Vertical partitions provide information about activities with different duration. In this case, we have used as time references the usual distinction, in years and semesters, implemented in universities. The beginning/ending 


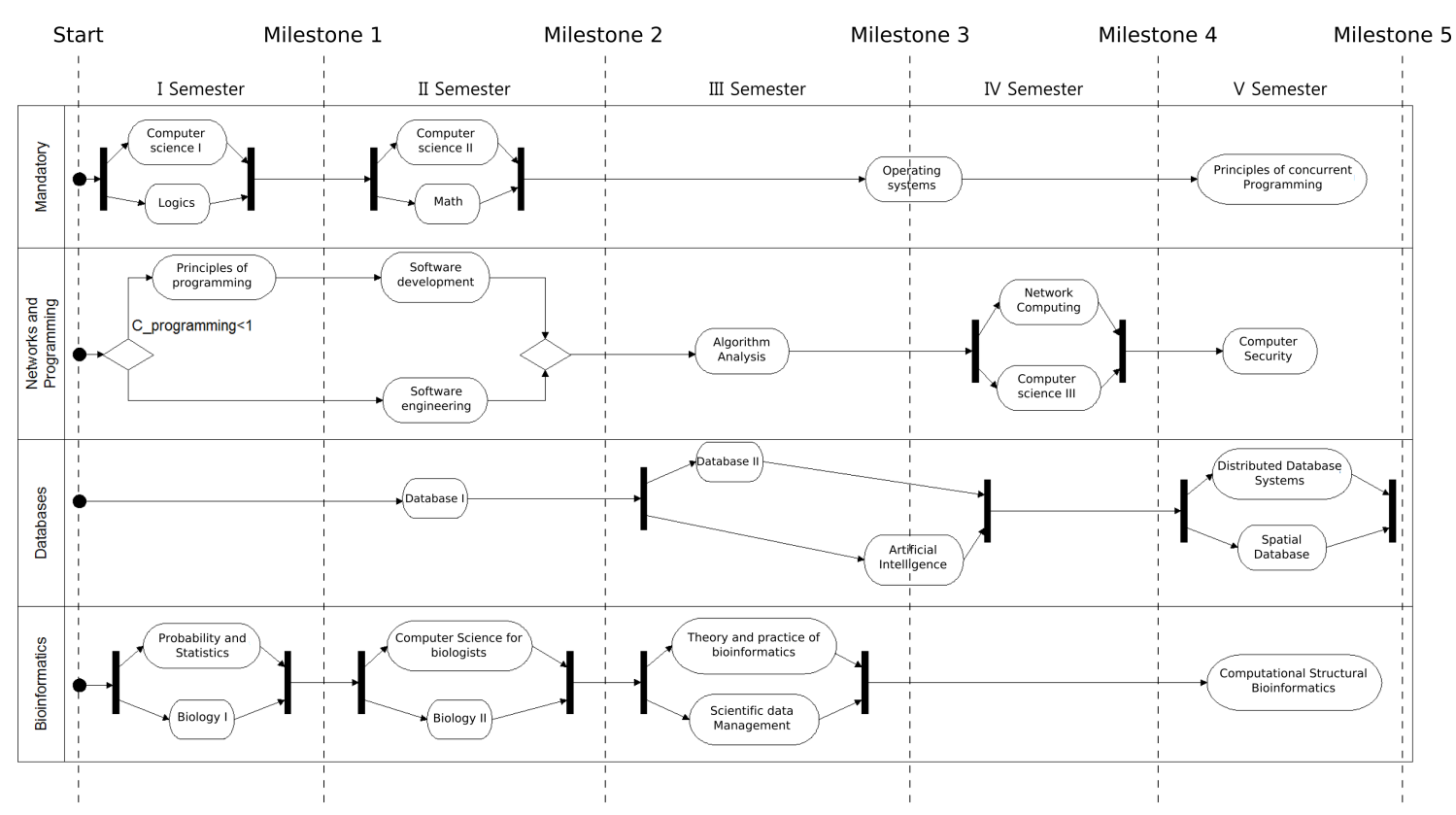

Figure 5: Activity diagram representing a curriculum with mandatory and additional, student chosen, courses. Swimlanes represent the sequencings of courses. Vertical divisions capture the different milestones (semesters).

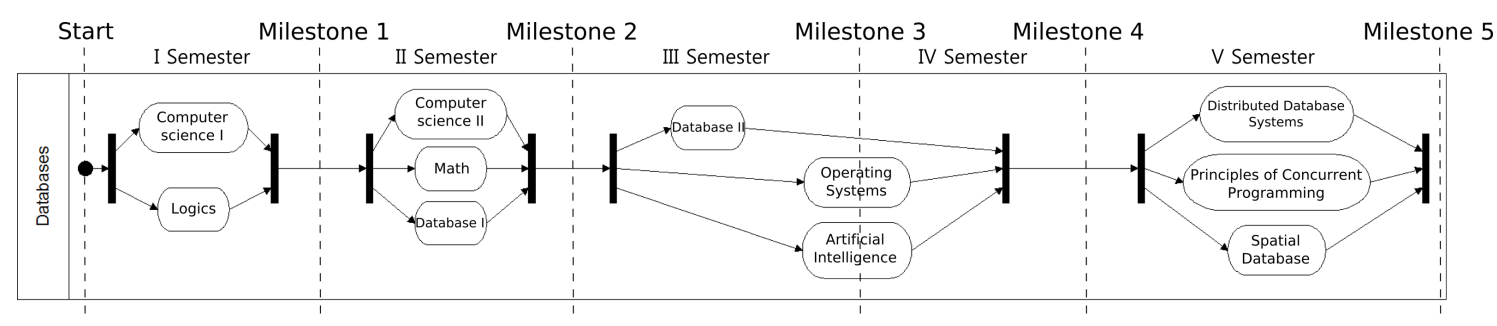

Figure 6: Example of a possible pathway obtained from the curriculum in Figure 5 by merging the mandatory pathway with the Databases additional pathway.

points of the semesters correspond to a set of milestones: this temporal organization will be used to identify those states, at which the verification will be applied (see below for an example). In previous works $[46,11]$, instead, courses were atemporal and each state was tied to the simulation of a single course. The introduction of durations allows a more realistic representation of the curricula and, especially, of the dependencies between competences. Therefore, we can easily see that the course Logics (in the mandatory pathway) is delivered during the first semester, while the course Operating Systems (in the same pathway) is delivered in the third and fourth semesters.

The swimlanes that represent additional learning paths can be used to capture one-time choices of the learners. Due the semantics of UML activity diagrams, each learner has to choose one of the optional learning paths in order to complete its curriculum ${ }^{3}$. For instance, once the learner has decided to become "database specialist", he/she has to complete the mandatory pathway, by merging it with the process represented in the swimlane Databases. Figure 6 shows the curriculum resulting by merging the mandatory learning path and the Databases learning path in Figure 5 . It

\footnotetext{
${ }^{3}$ Notice that it is possible to include an empty swimlane when the learner has the right of ignoring all of the optional pathways.
} 
should also be noted that processes representing a curriculum are only views combining activities. This is very compatible with UML activity diagrams where it is possible to reuse, in distinct contexts, activities defined once.

Another case to consider is when some two-semester course overlaps in time with another course. With reference to our example, Figure 5 Databases swimlane, the course Artificial Intelligence spans over the third and the fourth semesters, partially overlapping with the Database $I I$ course (held in parallel in the third semester). We cannot expect that Database II supplies competences that are preconditions to Artificial Intelligence. This issue can be solved once again by exploiting UML partitions. In fact, time overlapping can be handled by regulating the size and the relative position of the vertical partitions, i.e. by using milestones. The "timed semantics" remains underspecified and may be approached in the classical way by introducing time-dependent constraints on activity edges (or, on top of the interpretation of the UML superstructure specification - that often does not provide a sufficient level of detail - constraints attached to the partitions themselves).

\section{$5 \quad$ Validation of curricula}

The kinds pedagogical validation foreseen by the proposed authoring architecture are inter-conceptual and performed after the construction of a curriculum. In other terms, given a curriculum expressed as a UML activity diagram, it is possible to verify that all of its possible executions:

1. allow the achievement of the user's learning goal, contained in the learner model;

2. do not show any competence gap w.r.t the resource model;

3. satisfy the constraints in the curricula model.

All these validation tasks have been implemented by exploiting model checking techniques. Model checking is normally use to verify the properties of system. Two elements are needed: the set of facts that one wants to verify and the relevant aspects of the system that are needed to verify those facts [40]. In our case, the system is the curriculum, while the facts to verify are respectively given by the learner model, the resource model or the curricula model, depending on the validation at issue. SPIN, by G. J. Holzmann [40], is one of the best known tools of this kind, for LTL formulas. In order to perform the validation tasks, our proposal is to use SPIN, to translate the UML activity diagram, that encodes the curriculum, in a Promela program (where Promela is the language used by SPIN), and, then, to verify whether this program satisfies the LTL formulas which encode the facts to prove. We perform the three validation tasks at the same time.

The use of SPIN is orthogonal to the semantics of LTL. Any model checker for this logic or any optimized algorithm that allows the same verifications could be used instead of it. This is the advantage of adopting a declarative semantics for the curricula model. The important thing is that the implementation is proved sound w.r.t. the LTL semantics. The choice of SPIN is due to the fact that SPIN can tackle any LTL formula, and this gave us some freedom while we were defining the semantics of the various DCML operators because we did not have to modify in any way the model checker. Moreover, when a model does not satisfy some LTL formula (or assertion), SPIN produces a counterexample that shows the violation. This is very important in order to return feedbacks to the users, that help in correcting curricula (or curricula models).

Let us now introduce how a UML activity diagram, that expresses a curriculum, can be translated into a Promela program. In the literature, we can find some proposals to translate UML activity diagrams into Promela programs, such as $[32,35]$. These proposals have a different purpose than ours and they cannot directly be used to perform the translation that we need to perform the verifications we list above, however, it is possible to follow them as guidelines to perform our translation. Generally, their aim is debugging UML designs, by helping UML designers to write sound diagrams. The translation proposed in the following, instead, aims to simulate, by means of a Promela program, the acquisition of competencies by attending courses contained in an activity diagram. The verification process is, in a way, a simulation of the learning paths that can be 
obtained by combining the mandatory and the optional swimlanes in the UML activity diagram. Every milestone corresponds to a state, containing specific competences - all those acquired up to that point, partly because belonging to the learner from the very beginning and partly because achieved by attending courses. The initial state contains all the facts concerning the user at issue, which are contained in the learner model. The final state contains the competences that are gained by using the various learning resources which lay upon the followed learning path.

We represent all the competences involved by a UML activity diagram as integer variables. In the beginning, only those variables that represent the initial knowledge owned by the learner are set to a value greater than zero. Courses are represented as actions that can modify the value of such variables. Since we suppose that the learner's knowledge can only grow, also the value of variables can only grow.

The Promela program produced by the translation simulates the way competences are acquired, updating the set of the achieved competences at every step. Steps correspond to the various milestones into which the curriculum is organized. For instance, in Figure 5 we identify the initial state, a second state corresponding to the end of the first semester, another corresponding to the end of the second semester, and so on, up to a final state, corresponding to the end of the curriculum $^{4}$. Hereafter, we report the top level of the program corresponding to Figure 5:

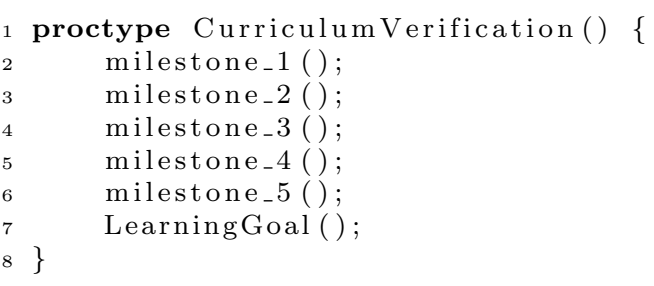

In order for the curriculum to contain no competence gap, it is sufficient that all the milestone procedures are completed successfully. In order to understand how milestones are implemented, let us see, first, how courses are encoded. Each course is represented by its preconditions and its effects. For example, the course Database_II is encoded as follows:

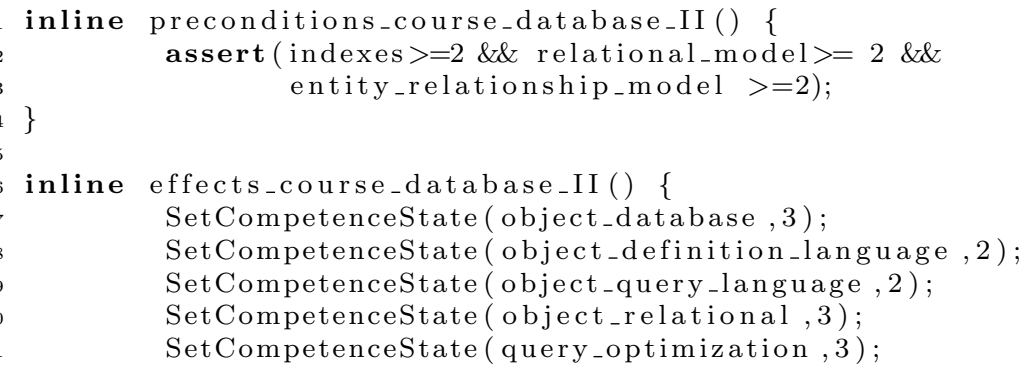

The SPIN instruction assert verifies the truth value of its condition, which in our case is the precondition to the course. If violated, SPIN interrupts its execution and reports about it. SetCompetenceState is used in the implementation of the effects of a course: it affects the current state, by adding new competences or by increasing the level of proficiency of some competence. If all the learning paths represented by the translated UML activity diagram have no competence gap, no assertion violation will be detected. Otherwise, a counterexample will be returned that corresponds to a sequence of courses bringing about the violation, thus, giving a precise feedback to the student/teacher/instructional designer.

Generally speaking, a milestone implements a transition in the learning process where: (1) all the preconditions of the courses attended in that phase are checked; (2) all the effects of courses attended in that phase are added to the state. If some preconditions are not satisfied a competence gap is identified. If the curriculum contains various possible learning paths, every milestone verifies

\footnotetext{
${ }^{4}$ In this article we report only some parts of the Promela program obtained by translating the example in Figure 5. The whole program, as well as the LTL formulas corresponding to the whole curricula model in Figure 2, are available at http://www.di.unito.it/ emarengo/DCMLexamples.
} 
the mandatory courses and simulates all of the different alternatives concerning optional courses. This is done by means of the introduction of an array of variables, that is used to discriminate among the alternative paths.

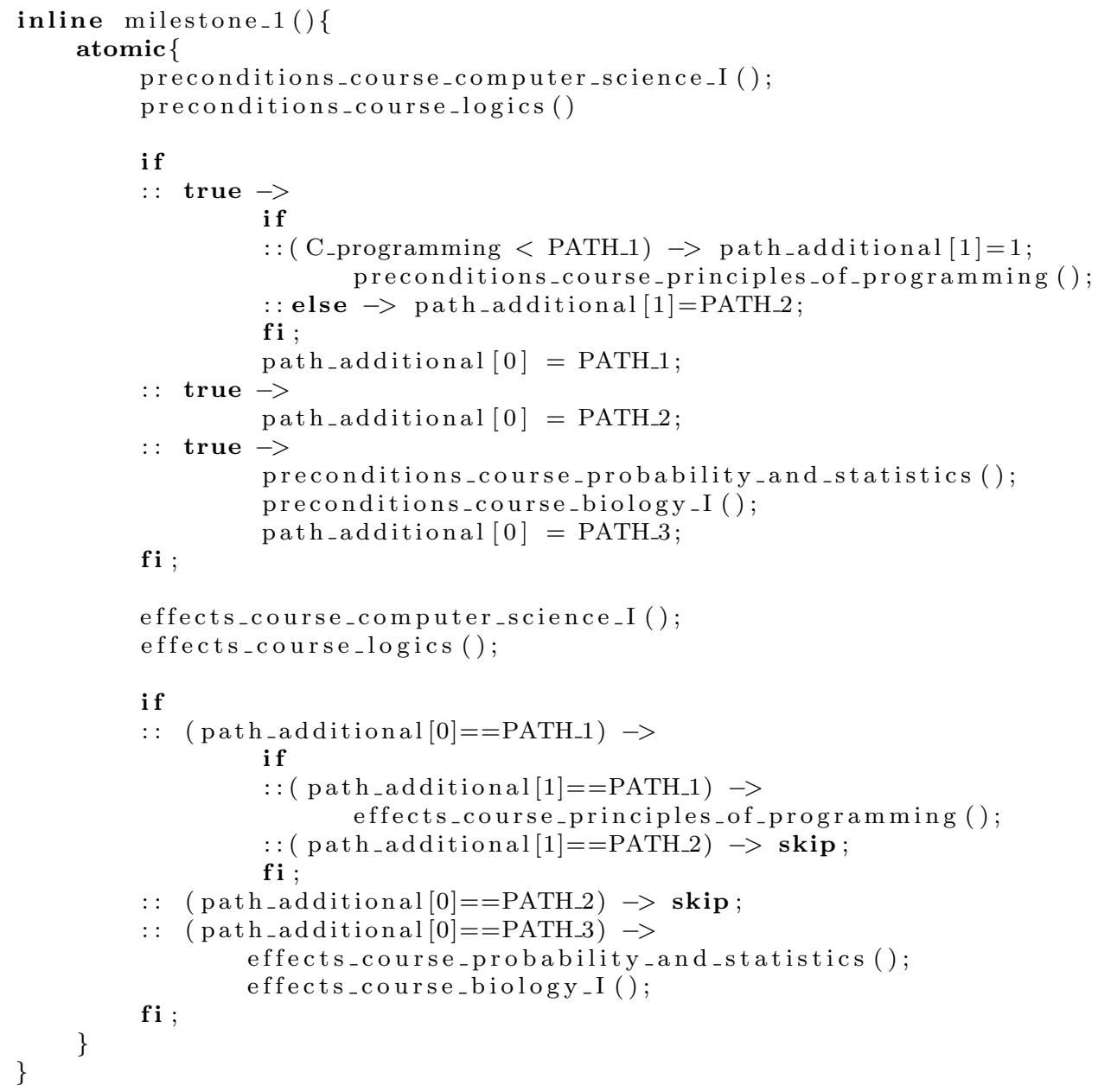

The last instruction of the process Curriculum Verification, which is applied only if the curriculum can be executed to its end, is LearningGoal. LearningGoal checks whether the user's learning goal is satisfied, by applying a test on the knowledge in the final state. For example, a learner interested in event-driven programming, concurrent programming, and file systems could have the goal:

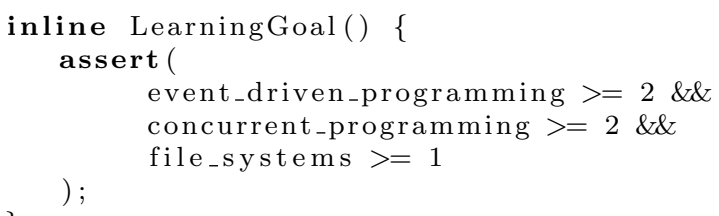

To check if the curriculum complies to a curricula model, first, the curricula model is turned into the corresponding LTL formulas, by applying the translations given in Section 3. Notice that SPIN uses strong until " $U$ " and not weak until "U", as we have done, therefore, we use the equivalence relation $\phi \cup \psi \equiv(\phi U \psi) \vee \square \phi$. Then, it is possible to apply SPIN and check if every possible learning path satisfies them. Since all the DCML constraints are in conjunction with each other, it is possible to check them one at a time, thus reducing the complexity of the problem drastically, because the automaton resulting for a single constraint is small. As soon as a constraint verification fails, the procedure stops. 


\section{Implementation in the Personal Reader Framework}

We designed the Personal Reader (PR) Framework ${ }^{5}$ as a tool and test-bed for creating applications in the Semantic Web. It offers an environment for designing, implementing and realizing Web content readers using a service-oriented approach, for a more detailed description, see [38]. In applications based on the Personal Reader Framework, users can select and combine - plug together - the personalization support they want to receive. The framework has already been used for developing Web Content Readers that present online materials in an embedded context $[16,3,37]$.

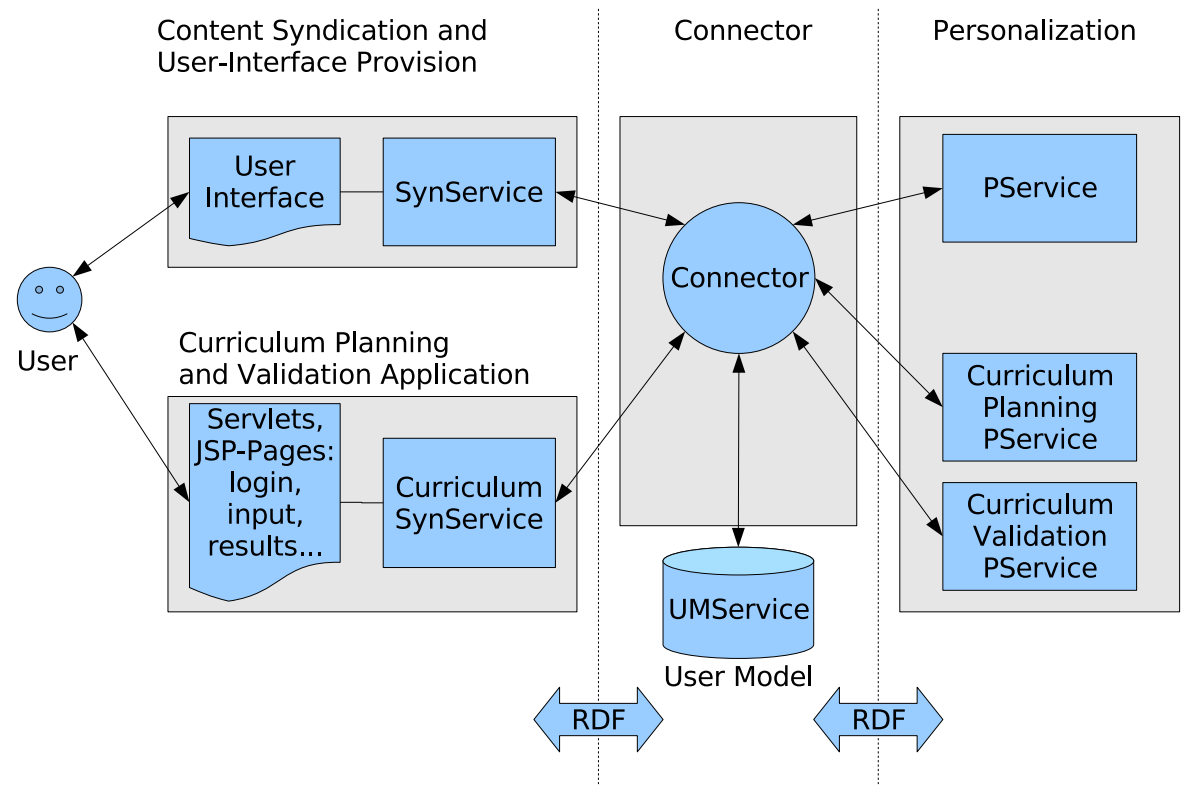

Figure 7: Personal Reader Framework Overview.

Figure 7 gives a brief overview of the main components of the PR architecture. A typical PR application consists of three types of services. Personalization services (PService) provide personalization functionalities: they deliver personalized recommendations for content, as requested by the user and obtained or extracted from the Semantic Web. The Syndication Services (SynService) realize the user interface and facilitate interoperability with the other services in the framework, e.g. it allows for the discovery of the applications' interfaces by a portal. The Connector is a single central instance responsible for controlling the communication between user interface and personalization services. It selects services based on their semantic description and the requirements by the SynService. The Connector protects - by means of a public-key-infrastructure (PKI) - the communication among the involved parties. It also supports the customization and invocation of services and interacts with a user modelling service, called the UMService, which maintains a central user model.

The Personal Reader architecture requires web services to be described in OWL-S, and also exploits taxonomies [3] for describing services, their capabilities and their configurable options (required for personalization). This information is used for accomplishing the service selection task by the Connector (see Figure 7). Notice that the aims of the PR are, however, different than those of frameworks like WSMO [53] and IRS-III [1]: the focus, in fact, is not to provide a generic framework to create web service compositions but to configure web services according to user and application requirements, and select services based on their personalization functionality.

${ }^{5}$ http://www.personal-reader.de/ 
For the implementation of the Curriculum Planning and Validation Application, the User Interface (SynService) was realized using Servlets and JSP Pages. Other applications, like the MyEar system [38] also use active scripting approaches (Java Script and Ajax) for creating adaptive user interfaces. For the actual data processing and personalization, two PServices were implemented, one for performing the curriculum sequencing task, the other hosting the validation engine.

\subsection{Metadata Description of Courses}

To apply our ideas to a real world scenario, we created the resource model (a corpus of courses) by extracting information from an existing database. We used the Lixto [15] tool to extract the needed data from the web-pages provided by the HIS-LSF ${ }^{6}$ system of the University of Hannover. This approach was chosen based on our experience with Lixto in the Personal Publication Reader [16] project, where we used Lixto for creating the bibliographic database by crawling the publication pages of all the REWERSE partners. The use of Lixto is motivated by the fact that a lot of information about courses and academic activities is already on-line, though its representation does not include a semantic annotation and it is unlikely that such an annotation will be added manually in the future. Hence the need of adopting a tool that is capable of extracting such a semantic annotation automatically. The effort to adapt our existing tool for the new data source was only small. From the extracted metadata we created the RDF knowledge base. For each of the courses, the course name, catalog identifier, semester, the number of credit points, effects and preconditions, and the type, e.g. laboratory, seminar, or regular course with examinations in the end, was recorded. Figure 8 shows the metadata properties of the course Digital Image Processing.

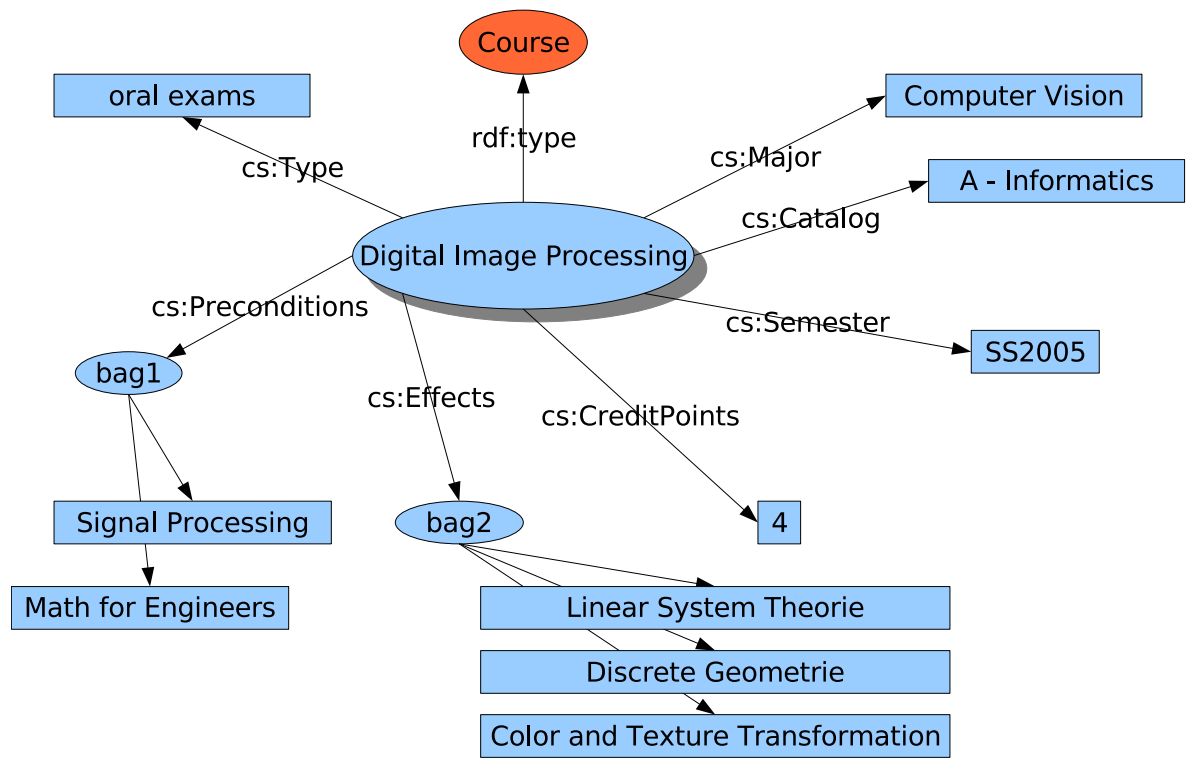

Figure 8: Metadata for the course Digital Image Processing from the Hannover course database.

As it turned out, the biggest problem was that the quality of most of the information in the database is insufficient, largely because of inconsistencies in the description of prerequisites and effects of the courses. Additionally the corpus was not annotated using a common set of terms, but course each author and department secretary used a slightly varying vocabulary in the descriptions that they edited, instead of relying on a common classification system, like e.g. the ACM Computing Classification System (ACM CCS ${ }^{7}$ ).

${ }^{6}$ http://www.his.de/

${ }^{7}$ http://www.acm.org/class/ 
As a consequence, we focussed only on a subset of the courses (computer science and engineering courses), and manually post-processed the harvested data. In our system, courses are annotated with prerequisites and effects, which correspond to either required or supplied competences. After the automatic extraction of effects and preconditions, the collected terms were spell-checked and harmonized, synonyms were removed and annotations were corrected where necessary. The resulting corpus had a total of 65 courses left, with 390 effects and 146 preconditions.

\subsection{The User Interface and Syndication Service}

The Syndication service (SynService) is responsible for creating the user interface to present to the learner, for requesting the necessary personalization functions from the connector, as well as all for the communication between the different Web services. In particular, it identifies the user and presents him/her an interface that allows the selection of the competences to be acquired. Furthermore, it has to display the results of the planning and validation processes (see Figure 9), allowing for further refinement of the created plans.

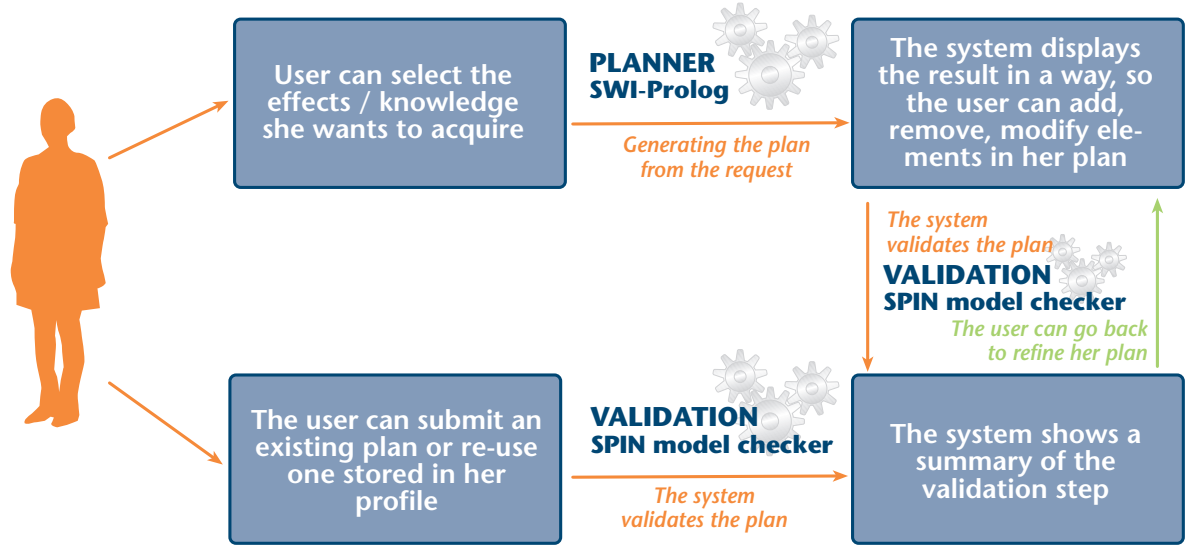

Figure 9: The Actions supported by the User Interface.

The curriculum sequencing and the validation tasks are delegated to two independent Personalization Services, the Curriculum Planning PService, and the Curriculum Validation PService. Because of the Plug \& Play nature of the infrastructure, the two PServices can be used by other applications (SynServices) as well (Figure 9). It is also possible to use additional PServices by extending the SynService so to provide additional planning and validation capabilities to our application. The current implementation of the Curriculum Planning and Validation Prototypes can be reached via the Projects page of the Personal Reader Homepage http://personal-reader.de. Figure 10 is a screenshot of the initial learning goal selection page, as generated by the syndication service and presented to the user. It contains fields for up to three required goals, as well as the number of credit points to achieve and the number of plans to generate.

\subsection{Automatic construction of curricula: the Curriculum Planning PSer- vice}

We have developed a simple service for building personalized curricula, which has been integrated as a Plug \& Play personalization service in the Personal Reader architecture. The curriculum is personalized in the sense that it allows a user to reach his/her learning goals, starting from the current competences the user has, which are included in the user model. 


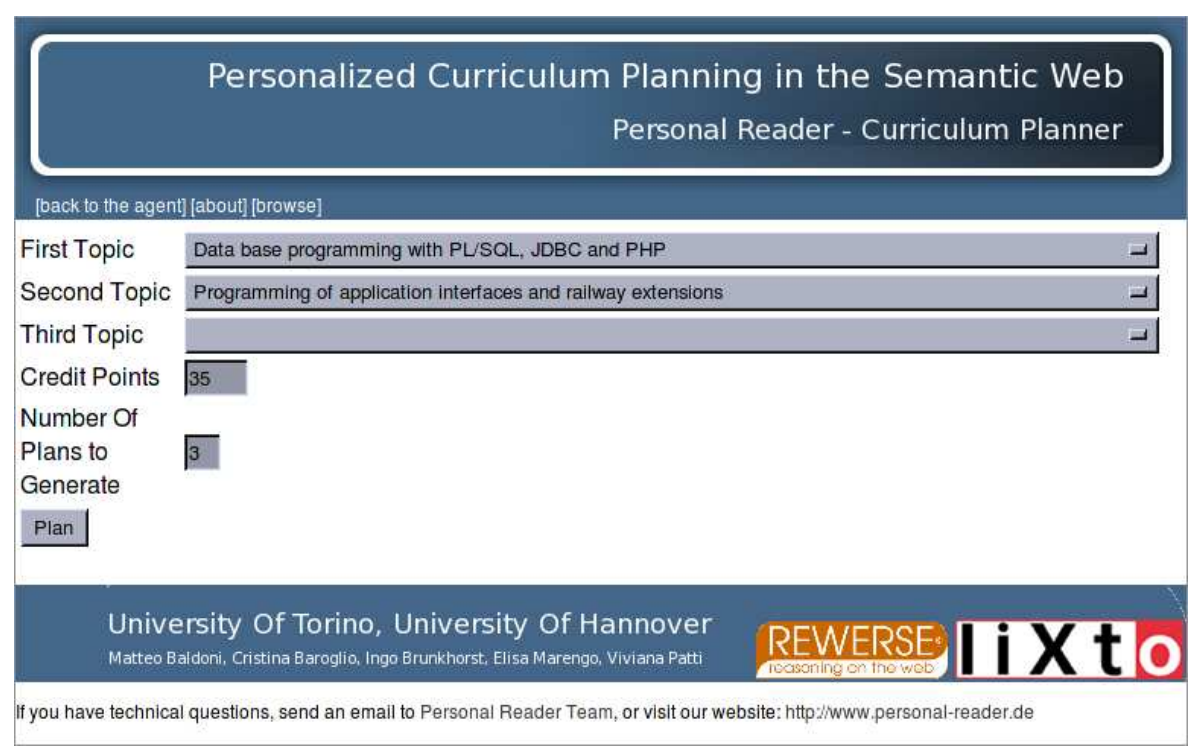

Figure 10: Selection of Learning Goals.

The planner was implemented as a Prolog program, so we had to embed a Prolog reasoner into the Web service. The planner executes a simple depth-first forward planning (an early prototype was presented in [7]), where actions cannot be applied more than once. The algorithm is simple:

1. starting from the initial state, the set of applicable actions (those whose preconditions are contained in the current state) is identified;

2. one of such actions is selected and its application is simulated leading to a new state;

3. the new state is obtained by adding to the previous one the competencies supplied as effects of the selected action;

4. the procedure is repeated until either the goal is reached or a state is reached, in which no action can be applied and the learning goal is not satisfied;

5. in the latter situation, backtracking is applied to look for another solution.

The procedure will eventually end because the set of possible courses is finite and each is applied at most once (the trivial assumption is that a course can be attended only once). If the goal is achieved, the sequence of courses that label the transitions leading from the initial to the final state is returned as the resulting curriculum. If desired, the backtracking mechanism allows the collection of a set of alternative solutions to present to the user.

Figure 11 gives an overview over the components in the current implementation. The Web service implements the Personalization Service (PService for short, see [38]) interface, defined by the Personal Reader framework, which allows for the processing of RDF documents and for inquiring about the services capabilities. The Java-to-Prolog Connector runs the SWI-Prolog executable in a sub-process; essentially it passes the RDF document containing the request as-is to the Prolog system, and collects the results, already represented as RDF.

The curriculum planning task itself is accomplished by a reasoning engine, which has been implemented in SWI-Prolog ${ }^{8}$. The interesting thing of using SWI-Prolog is that it contains a semantic web library allowing to deal with RDF statements. Since all the inputs are sent to the reasoner in a $R D F$ request document, it actually simplifies the process of interfacing the planner

\footnotetext{
${ }^{8}$ http://www.swi-prolog.org/
} 


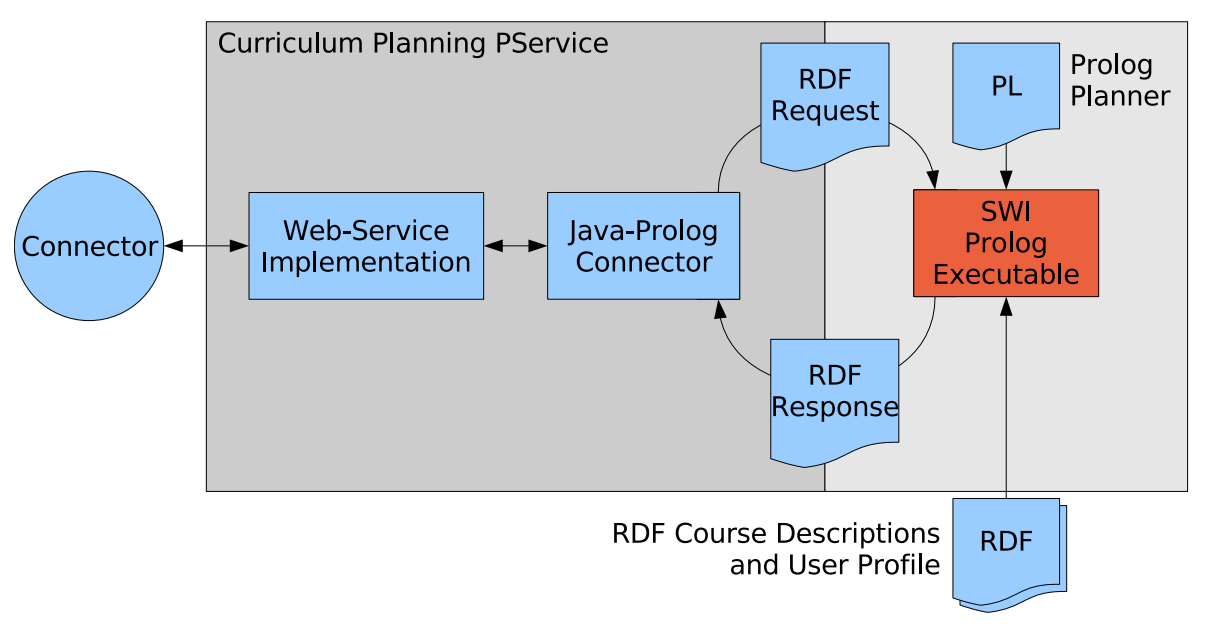

Figure 11: Curriculum Planning Web Service.

with the Personal Reader. In particular the request document contains: a) links to the RDF document containing the database of courses, annotated with metadata (the resource model), b) a reference to the learner model, including the user's actual learning goal, i.e. a set of competences that the learner would like to acquire, and that concern competencies belonging to the domain model. The reasoner can also deal with information about credits provided by the courses, when the user sets a credit constraint together with the learning goal.

At the end of the process, a $R D F$ response document is returned as an output. It contains a list of plans (learning paths) that fulfill the learner model. The maximum number of possible solutions can be set by the learner in the request document. Notice that further information stored in the learner model is used at this stage for adapting the presentation of the solutions, here simple hints are used to rank higher those plans that include topics for which the user has expressed a special interest. Figure 12 shows the output generated by the syndication service for the generated plan, decoded from the RDF response document. The interface also provides the means for the learner to modify the plan and submit it for further validation.

\subsection{The Curricula Validation PService: current state of the implemen- tation}

Given a curriculum, it is possible to perform post-construction validation tasks in order to verify if the curriculum allows the achievement of the learning goal, if it contains competence gaps, and if it satisfies the constraints of a given curricula model, by following the process described in Figure 13. Once a curricula model is defined, it can be translated into a LTL formula by following the process described in Section 5.

For what concerns the interaction of the learner with the validation system, there is the need of allowing the learner to insert the curriculum to be validated into the system. The easiest way for allowing an a naïve user, like the average student, to perform this task is to ask him/her to enter a sequence of courses, corresponding to the desired curriculum, by using a web interface (as it was done, for instance, in the WLog system [13]). A linear plan is the simplest kind of curriculum that can be captured by means of an activity diagram, however, this choice makes sense because it is unlikely that the student learns to exploit the full potential of an activity diagram-based representation in order to express curricula. A good handling of activity diagrams requires, in fact, some expertise that the naïve user does not have. Moreover, since the planning PService produces linear plans, it is also possible to compose the effect of the planning process with the validation service, in order to check the compliance of an automatically generated curriculum with 


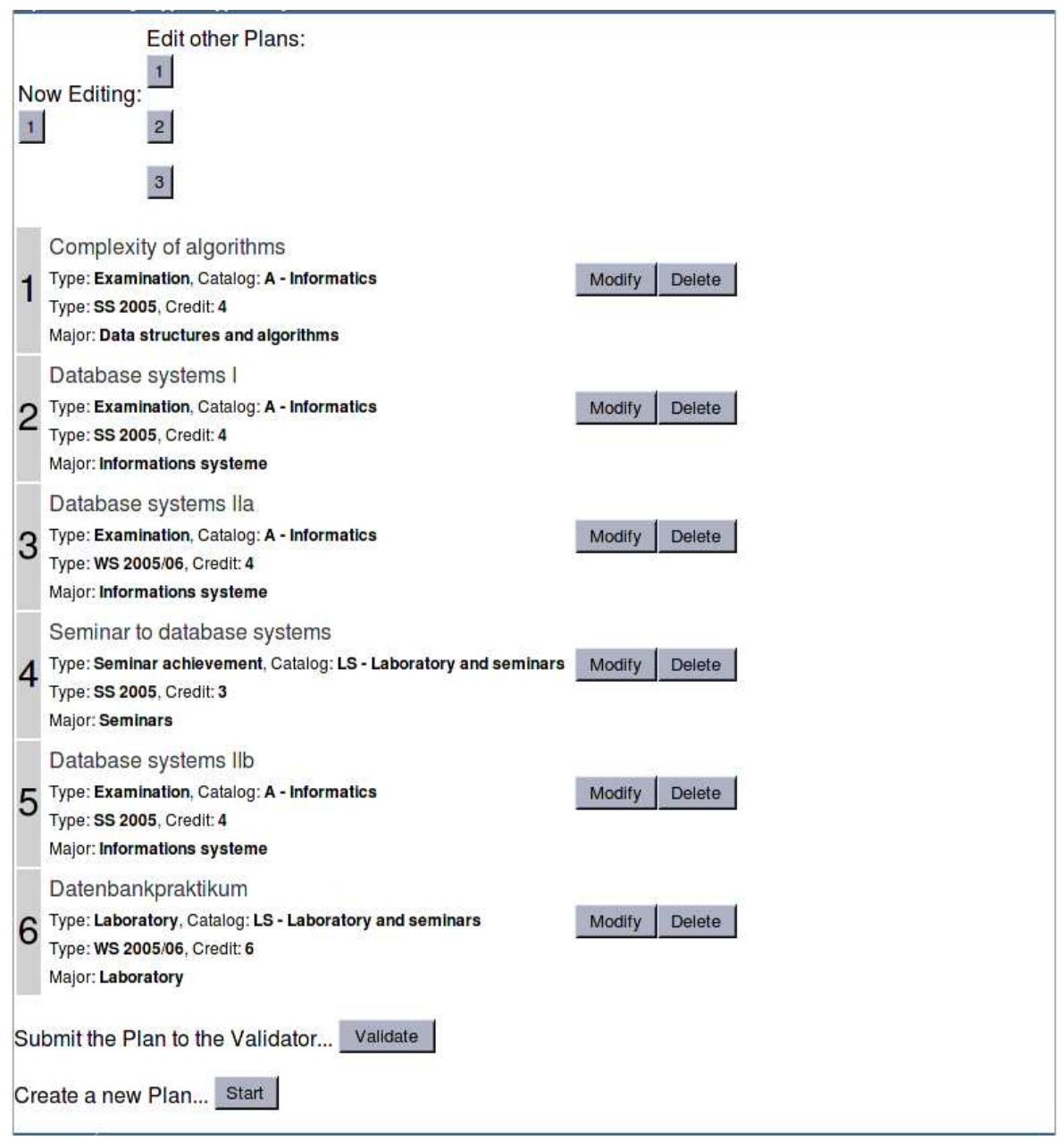

Figure 12: Output of the Planning Step.

a given curricula model.

The full potential of activity diagrams can, instead, be exploited by the instructional designer, when he/she faces the task of building and proposing new educational paths, which may, for instance, include a mandatory part and one or more (alternative) options. In this case, the interaction will not necessarily be performed via the browser. The designer can, in fact, write activity diagrams by means of standard UML design system. Moreover, for helping the instructional designer in the construction of curricula models, we have developed an Eclipse plugin (the $D C M L$ Designer, see Figure 14 [51]). This tool is thought for being used by the instructional designers of an academic institute. The task of an instructional designer is to define the educational offer of the institute and the curricula models that must be respected by the curricula defined by the students.

In order to perform the check that a curriculum does not show competence gaps and supplies the user's learning goal, it is required to interface the Validation PService with the RDF course descriptions (contained in the resource model) and with the learner model. This can be done along the lines of what we have described in the previous section by translating a curriculum into Promela code. 


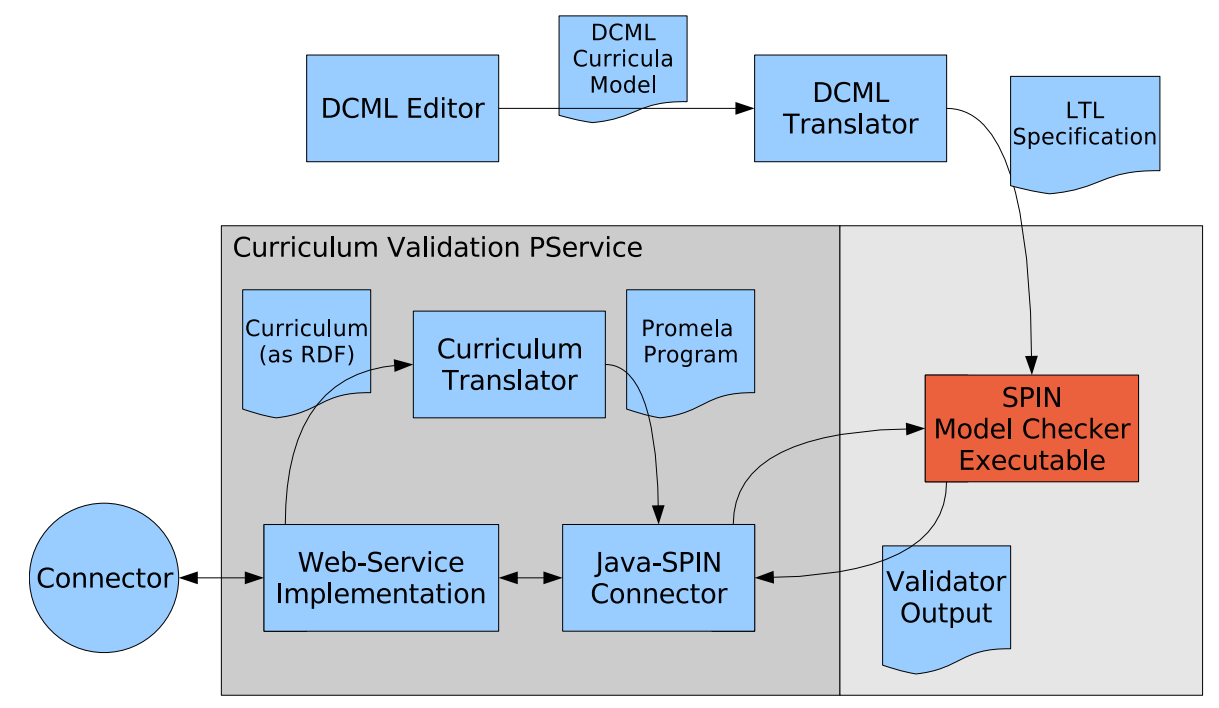

Figure 13: The validation w.r.t. a curricula model: process workflow.

\section{Extending DCML to deal with time proximity}

The constraints presented in Section 3 express temporal relations which do not capture the time proximity between the acquisitions of two competences. It is often the case, however, when by saying that a competence is to be acquired, for instance, before another competence, the designer actually means "immediately before", e.g. in the previous semester. In order to express this stronger kind of relations we have extended the core of DCML by adding the notions of immediateness. All the relations that we have introduced (see Figure 4) have a correspondent stronger version: immediate before, immediate implication, immediate succession and their negations. Graphically, the notion of immediateness is represented by double arrows (see Figure 15). In the following we briefly introduce these new relations, whose definitions exploit the temporal logic operator next-time: $\bigcirc \varphi$ means that the formula $\varphi$ holds in the next state of the run.

\section{Immediate before}

Immediate before (see Figure 15) is represented by means of a double line arrow that ends with a little-ball. The constraint $\left(k_{1}, l_{1}\right)$ immediate before $\left(k_{2}, l_{2}\right)$ imposes that $\left(k_{1}, l_{1}\right)$ holds before $\left(k_{2}, l_{2}\right)$ and the latter either is true in the next state w.r.t. the one in which $\left(k_{1}, l_{1}\right)$ becomes true or $k_{2}$ never reaches the level $l_{2}$. The difference w.r.t the before constraint is that it imposes that the two competences are acquired in sequence. The corresponding LTL formula is " $\left(k_{1}, l_{1}\right)$ before $\left(k_{2}, l_{2}\right) " \wedge \square\left(\left(k_{1}, l_{1}\right) \supset\left(\bigcirc\left(k_{2}, l_{2}\right) \vee \square \neg\left(k_{2}, l_{2}\right)\right)\right)$. More generally, in presence of DNF formulas as antecedent and consequence of a "immediate before" relation, we have the following definition for "dnf $f_{1}$ immediate before $d n f_{2} "$ :

$$
\bigvee_{c f_{i} \in d n f_{1}, c f_{j} \in d n f_{2}} " c f_{i} \text { before } c f_{j} " \wedge \square\left(c f_{i} \supset\left(\operatorname{next}\left(c f_{j}\right) \vee \text { absence }\left(c f_{j}\right)\right)\right)
$$

where $\operatorname{next}(c f)=\bigwedge_{\left(k_{i}, l_{i}\right) \in c f} \bigcirc\left(k_{i}, l_{i}\right)$.

Example 7.1 As an example, with reference to Figure 2 - right, let us consider the constraint: (data_transfer, 1) immediately before (classical_encryption, 2). If the competence (data_transfer, 1 ) is acquired, then this must happen in the state that precedes the one in which (classical_encryption, 2) is acquired.

This kind of constraint is useful because, even though we assume that once a competence is acquired, then, it cannot be removed from the set of competences owned by the student, in real life 


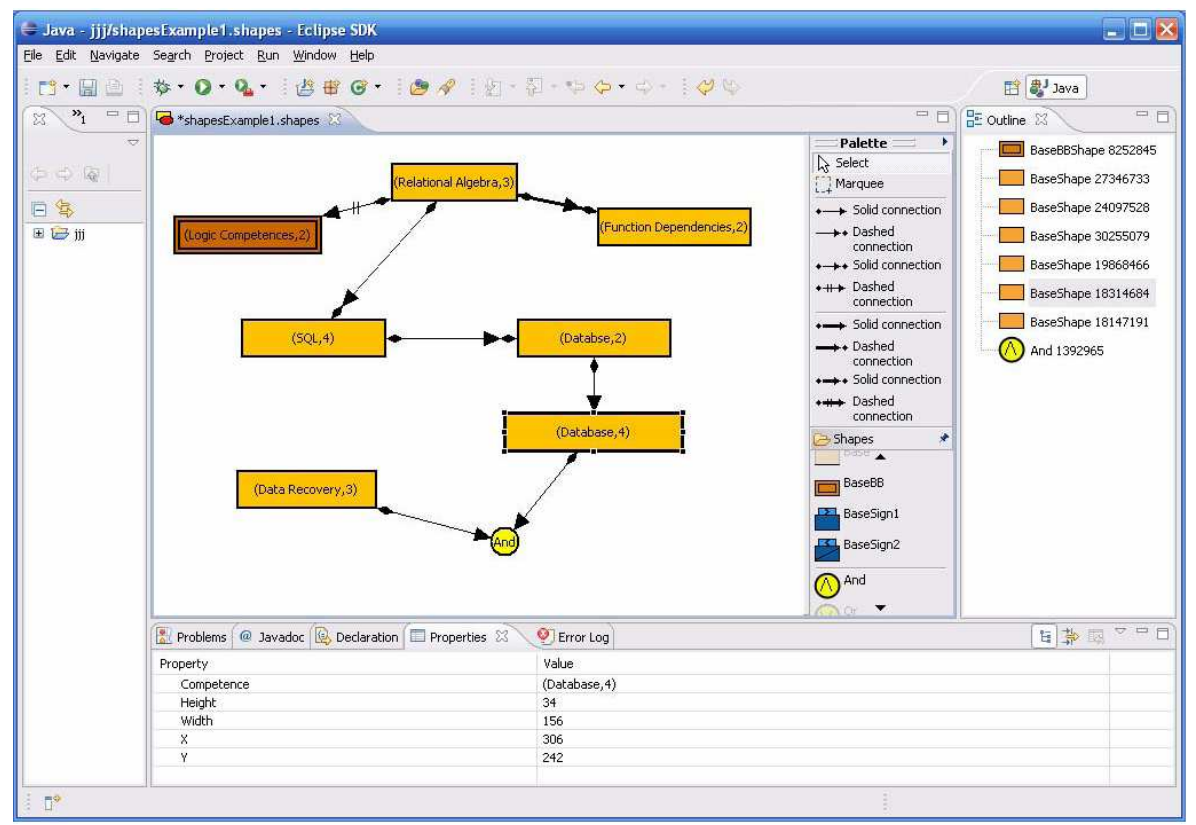

Figure 14: A screenshot of the DCML designer.

students tend to forget part of what they learnt. In some cases, however, it is important to be sure that certain competences are owned in (almost) their integrity in order to allow the acquisition of some other competence. In this case, it is better to use the immediately before constraint rather than the before constraint.

The not immediate before is translated exactly in the same way as the not before. Indeed, it is a special case because we assume that a competence cannot be forgotten. More generally, in presence of DNF formulas, "dnf $f_{1}$ not immediate before $d n f_{2}$ " is:

$$
\bigvee_{c f_{i} \in \operatorname{dn} f_{1}, c f_{j} \in d n f_{2}} \text { negation }\left(c f_{i}\right) \cup\left(c f_{j} \wedge \text { negation }\left(c f_{i}\right)\right)
$$

\section{Immediate implication}

The immediate implication, instead, specifies that the consequent must hold in the state right after the one in which the antecedent is acquired. Note that this does not mean that it must be acquired in that state but only that it cannot be acquired afterwards. This is expressed by the LTL implication formula in conjunction with the constraint that whenever $k_{1} \geq l_{1}$ holds, $k_{2} \geq l_{2}$ holds in the next state: $\diamond\left(k_{1}, l_{1}\right) \supset \diamond\left(k_{2}, l_{2}\right) \wedge \square\left(\left(k_{1}, l_{1}\right) \supset \bigcirc\left(k_{2}, l_{2}\right)\right)$. More generally, in presence of DNF formulas as antecedent and consequence of a "immediate implication" relation, we have the following definition for " $d n f_{1}$ immediate implication $d n f_{2}$ ":

$$
\bigvee_{c f_{i} \in d n f_{1}, c f_{j} \in d n f_{2}} \text { "cf } f_{i} \text { implies } c f_{j} " \wedge \square\left(c f_{i} \supset \operatorname{next}\left(c f_{j}\right)\right)
$$

Example 7.2 Figure 2 (top) reports the following example of immediate implication: (software_and_services_for-_biology_on_the_web, 3) immediately implies (visualizing_informations, 1). In this case if the learner does not own the competence (visualizing_informations, 1) yet, he/she must acquire it, and this acquisition must be performed immediately after software_and_services_for-_biology_on_the_web. The reason is that in this case knowledge about software_and_services_for-_biology_on_the_web is considered to be helpful in order to better understand visualizing_information. 


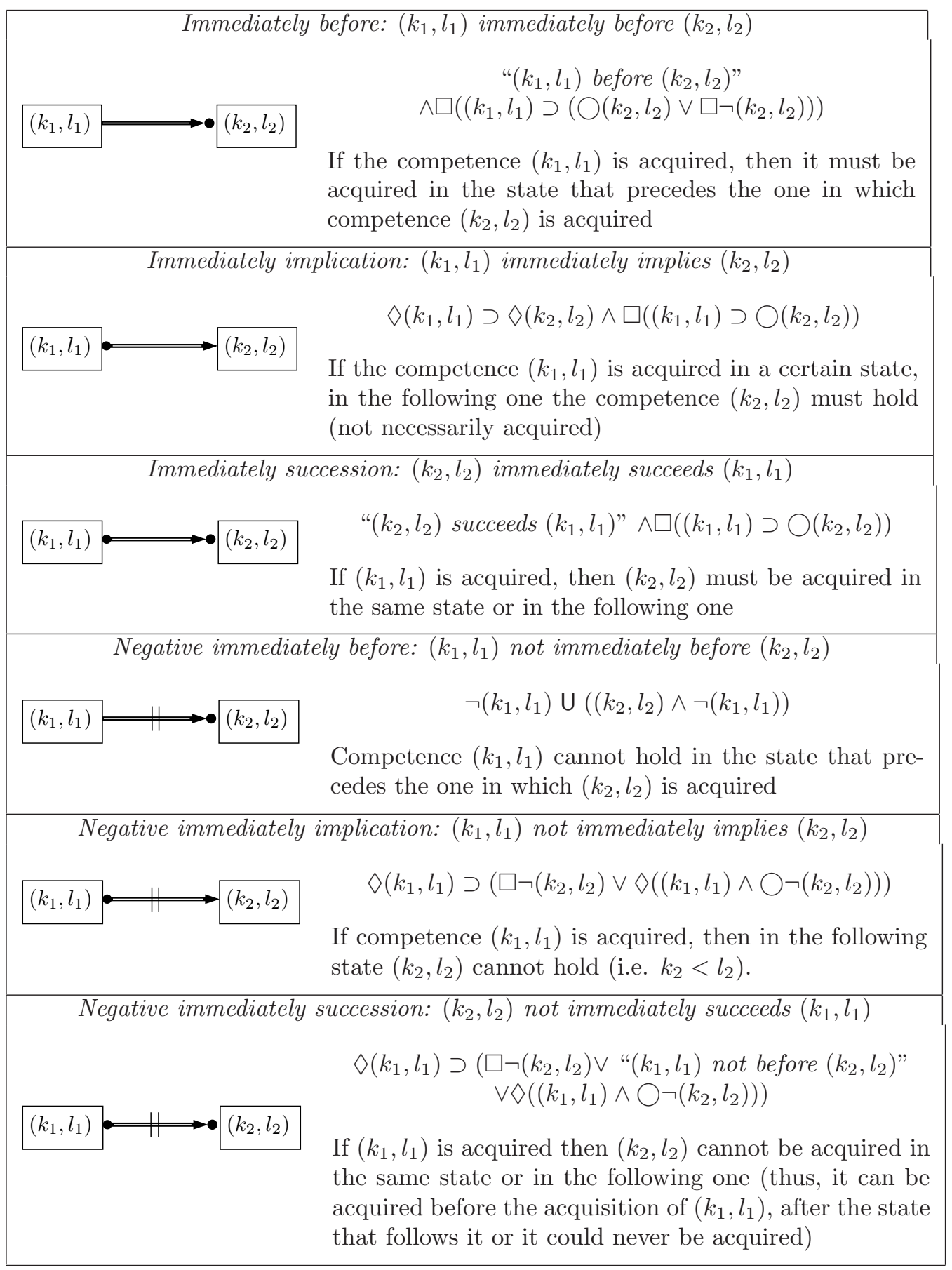

Figure 15: DCML notations for immediate before, immediate implication, immediate succession, and their negations. 
" $\left(k_{1}, l_{1}\right)$ not immediate implies $\left(k_{2}, l_{2}\right)$ " imposes that when $\left(k_{1}, l_{1}\right)$ holds in a state, $k_{2} \geq l_{2}$ must be false in the immediately subsequent state. Afterwards, the proficiency level of $k_{2}$ does not matter. The corresponding LTL formula is $\diamond\left(k_{1}, l_{1}\right) \supset\left(\square \neg\left(k_{2}, l_{2}\right) \vee \diamond\left(\left(k_{1}, l_{1}\right) \wedge \bigcirc \neg\left(k_{2}, l_{2}\right)\right)\right)$, that is weaker than the "classical negation" of the immediate implication. More generally, in presence of DNF formulas, " $d n f_{1}$ not immediate implies $d n f_{2}$ " is:

$$
\bigvee_{c f_{i} \in d n f_{1}, c f_{j} \in d n f_{2}} \operatorname{existence}\left(c f_{i}\right) \supset \text { absence }\left(c f_{j}\right)
$$

Example 7.3 Figure 2 (center) also reports an example of not immediately implies constraint: (query_optimization, 3) not immediately implies (distributed_query_optimization, 2).

This kind of constraint is useful to express the need of leaving some time to the learner, so that he/she can better assimilate some knowledge (about query_optimization in the example) before starting to face a complex topic (distributed_query_optimization).

\section{Immediate succession}

In the same way, the immediate succession imposes that the consequent either is acquired in the same state as the antecedent or in the state immediately after (not before nor later). The immediate succession LTL formula is " $\left(k_{2}, l_{2}\right)$ succeeds $\left(k_{1}, l_{1}\right)$ " $\wedge \square\left(\left(k_{1}, l_{1}\right) \supset \bigcirc\left(k_{2}, l_{2}\right)\right)$. More generally, in presence of DNF formulas as antecedent and consequence of a "immediate succession" relation, we have the following definition for " $d n f_{2}$ immediate succeeds $d n f_{1}$ ":

$$
\bigvee_{c f_{i} \in d n f_{1}, c f_{j} \in d n f_{2}} " c f_{j} \text { succeeds } c f_{i} " \wedge \square\left(c f_{i} \supset \operatorname{next}\left(c f_{j}\right)\right)
$$

Example 7.4 Figure 2 (top-center) shows the following example: (bioinformatics_web_based_resources, 1) immediately succeeds (software_and_services_for_biology_on_the_web, 3). As for succession, this constraint expresses a temporal ordering between the times at which competences are to be acquired (software_and_services_for_biology_on_the_web first). Moreover, it specifies that bioinformatics_web_based_resources is to be acquired either in the same state or in the state that immediately succeeds the one at which software_and_services_for_biology_on_the_web is acquired. Once again, the reason is that the instructional designer considers to be important that the learner retains in his/her mind all the knowledge acquired about the first topic, in order to understand the latter.

Its negation imposes that if a competence is acquired in a certain state, in the state that follows, another competence cannot be acquired, that is $\diamond\left(k_{1}, l_{1}\right) \supset\left(\square \neg\left(k_{2}, l_{2}\right) \vee\right.$ " $\left(k_{1}, l_{1}\right)$ not before $\left(k_{2}, l_{2}\right)$ " $\left.\vee \diamond\left(\left(k_{1}, l_{1}\right) \wedge \bigcirc \neg\left(k_{2}, l_{2}\right)\right)\right)$. Thus, when $\left.k_{1}, l_{1}\right)$ is acquired $\left(k_{2}, l_{2}\right)$ can already hold, it could never hold or it could be acquired in a state that follows the next one. More generally, in presence of DNF formulas, " $d n f_{2}$ not immediate succeeds $d n f_{1}$ " is:

$$
\begin{array}{ll}
\bigvee_{c f_{i} \in d n f_{1}, c f_{j} \in d n f_{2}} & \begin{array}{l}
\text { existence }\left(c f_{i}\right) \supset\left(\text { absence }\left(c f_{j}\right) \vee\right. \\
\left.\diamond\left(c f_{i} \wedge \operatorname{next}\left(\text { negation }\left(c f_{j}\right)\right)\right)\right)
\end{array}
\end{array}
$$

\section{Related works}

In the literature it is possible to find different works that are related to the different aspects of the approach we present here. A recent proposal for automatizing the competence gap verification is done by Melia and Pahl in [44], where an analysis of pre- and post-requisite annotations of the Learning Objects, representing the learning resources, is proposed. A logic based validation engine can use these annotations in order to validate the curriculum/learning object composition. Melia and Pahl's proposal is inspired by the CoCoA system, by Brusilovsky and Vassileva [21], that allows for the analysis and the consistency check of static web-based courses. Competence gaps are checked by a prerequisite checker for linear courses, simulating the process of teaching with an overlay student model. Pre- and post-requisites are represented as "concepts". In [46, 45], the same 
authors propose a layered model for pedagogical courseware validation (CAVaM or CAVIAr), that inspired our proposal. Two kinds of validation are identified: one against pedagogical strategies, the other against pedagogical rules. For what concerns the former, one pedagogical strategy is supported: checking for the presence of competency gaps. Instead, for what concerns the latter, the authors propose the use of a rule engine, JESS, in order to verify that learning objects are shaped in a way that satisfies the instructional designer. For instance, for all goal concepts there must be a learning object of type lecture which teaches it. With respect to Melia and Pahl's work, the goal and constraint model that we defined is richer, because it allows the representation of any proficiency levels, and the expression of a wider range of constraint. Namely, we can express before, implication, succession and their negations, as well as time proximity, while they only foresee one kind of constraint, the before (interpreted as a prerequisite). Moreover, our constraints have a declarative semantics given by LTL. For what concerns the architecture, we have added one more layer, explicitly representing resources based on the domain model. This layer allows the representation of constraints thare are local to a resource. Moreover, although they also use UML activity diagrams, they do not use the parallel operator, do not distinguish between mandatory and optional learning paths, and do not introduce the notion of duration of the composed activities. The last difference lays in the implementation of the validation tasks, that relies, in our case, in a model checker.

Along this line, also Garro et al. [33] propose an approach to the automatic construction of learning paths (analogous to curricula), that has been implemented as part of the MASEL system [34]. A learning path is a sequence of learning objects (analogous to learning resources), which allows the acquisition of a skill (a competence, in our terminology) that is desired by the user. Learning objects have a rich description; in particular, each learning object has some effects (skills that are supplied). Learning objects do not have preconditions, which are substituted by a "pre-requisite" relation between skills (this skill is to be acquired before this other skill). The approach also allows for the representation of the duration of learning objects and of the complementarity of certain sets of learning objects, moreover, skills include a proficiency level. In this work, however, the only reasoning task that is handled is planning, they do not have an explicit representation of curricula models and, therefore, they do not face the verification issues.

Brusilovsky and Vassileva [20, 21] adopt a method that is close, in principle, to curriculum sequencing: course sequencing. The aim of course sequencing, a technique originally proposed in the field of Intelligent Tutoring Systems, is to supply users with personalized courses, which select, at every step of the learning process, the best teaching method, i.e. the teaching method that will help the user to get the closest to his/her learning goal. The term "teaching method" refers, for instance, to the possibility of facing an exercise rather than reading more detailed documentation about a topic. In particular, two models are proposed: DCG and CoCoA. Both systems help the construction of personalized courses on the basis of a semantic network, which composes a set of Domain Knowledge Elements, roughly corresponding to our competences. DCG organizes such elements in an AND-OR graph, while CoCoA adopts an heterarchic structure relying on the relations part-of and attribute-of. Both organizations represent the domain model. DCG applies "dynamic planning" techniques: at every step the student's advancements are verified by a test. If the test is passed, a new topic is presented, otherwise some replanning is performed in order to allow the student to fill the gaps. Each node in the domain model can be presented in different ways (e.g. test, exercise, motivation, example). A presentation plan component has the task of identifying the best presentation of the concept for that particular student in that particular context. This selection is performed based on a set of "teaching rules" encoded in the system. Dynamic planning fits very well the task of building student-oriented personalizations but it does not scale equally well to class-based personalization unless all students in class have similar learning skills. CoCoA supports the construction of courses by performing a set of operations among which consistency checks and quality checks. Also in this case different kinds of presentation are identified. CoCoA can perform prerequisite checks by simulating the execution of a course and verifying at every step that the preconditions to the current step are satisfied, i.e. that at that point the student will have the necessary knowledge. Each kind of presentation of each concept has its own prerequisites (so there are question prerequisites, presentation prerequisites, etc.). A limit of this approach is 
that prerequisites allow the expression of a single kind of constraint: what is to be learnt before a certain learning step can be accomplished. We have shown, by presenting DCML, that there are many other kinds of relation that it is interesting to capture, leading to the introduction of a further level of abstraction: the one given by our curricula model.

Brusilovsky and Vassileva in [21] also define various verification tasks, besides competence gaps, among which two tasks that we accomplish in the present proposal: (a) verifying that the curriculum allows the achievement of the user's learning goals, and (b) verifying that the curriculum is compliant against the course design goals. Manually or automatically supplied curricula, developed to reach a learning goal, should match the "design document", a sort of curricula model, specified by the institution that offers the possibility of personalizing curricula. In [21] design documents are said to specify general rules for designing sequences of learning resources (courses). In this proposal, the design document has a procedural nature and the verification checks that the curriculum respects the defined structure. The authors, however, do not supply guidelines or specifications that help writing design documents. We interpreted such general rules not is a procedural manner but rather as constraints, that are expressed in terms of competences and, in general, are not directly associated to learning resources, as instead is done for pre-requisites. They constrain the process of acquisition of concepts, independently from the resources.

Another proposal is the one by Farrell, Liburd, and Thomas [31], who propose an approach called Dynamic Assembly. This approach allows the automatic generation of a course by composing pre-existing learning objects on the base of a set of parameters (e.g. level of detail, time, keywords) which are specified by the user. Parameters are expressed in a course assembly page. The system provides two methods, namely indepth and overview, which respectively narrow the search to the topics listed by the user and return also learning objects concerning related topics. The result of the search is a numbered sequence of links to learning objects. The approach does not supply methods for verifying that the produced sequences, besides reaching the goal, also "make sense" from an educational point of view, for instance by exploiting some abstract model.

The works in $[64,22,41]$ focus on the curriculum planning problem, intended as the problem of planning an academic schedule of activities. So the goal is to organize the different courses of an academic curriculum on a given set of time periods (e.g. semesters), satisfying some academic (e.g. course availability, prerequisites, eligibility rules) or student constraints. The problem of planning an academic schedule can be naturally tackled by adapting to the application context a Constraint Satisfaction Problem (CSP) modeling framework, as proposed in [64, 22, 41]. In general, CSP models can be considered a good starting point for dealing with bindings to the fruition of the learning resources that may depend on the kind of infrastructure supplied by the academic institution (e.g. number and location of rooms, support services for computing, number of teachers and so on). In particular, Wu and Havens [64] have proposed an extended model that exploits mixed-initiative constraint reasoning algorithms and provides flexibility in satisfying not only the constraints given by the institution offering the courses, but also the student's preferences and needs. In a first phase (curriculum planning), a set of courses available in a certain periodo of time is identified, by interpreting the task as a constraint satisfaction problem with preferences. Courses are then presented to the user by means of an interface that organizes them in a table, whose columns correspond to teaching periods. The user can adjust the presented solution by modifying part of it. The first plan is obtained by applying the constraint given by the institution, while the modification actions taken by the user are interpreted as user constraints. A modified plan is validated in order to see if it still respects the overall set of constraints and then the interaction with the user can be repeated. The kinds of constraint that the system can handle are of different types. Among them: a course can appear only once per plan, courses can require other courses (not competences) as prerequisites, it is not possible to attend "equivalent" courses, some courses are mandatory. As one can observe, this kind of constraints is not defined on the competences required/thought by courses nor they express the constraints on the fruition of the teaching materials supplied by the teacher.

In $[22,41]$ the authors tackle the problem of building balanced academic curricula. This term identifies curricula in which courses are well-distributed, according to a set of load constraints, 
along the trimesters/years. Besides load constraints also constraints concerning course prerequisites are considered during the planning phase. As in the previous approach, a course prerequisites correspond to other courses which are to be attended before the one at issue. The problem is interpreted as an optimization problem, which can be tackled in different ways (e.g. branch and bound, constraint programming, genetic algorithms).

Last but not the least, recently some attention has been posed also on ontologies for representing in a uniform way information like academic programs and examination regulations. For example, [36] proposes an ontology for representing this information as well as the universities supply of courses and the individual results of the students. The idea is to use this kind of ontologies in the process of producing or validating personalized curricula.

\section{Conclusions}

This article integrates and extends the results of a collaboration between the Department of Computer Science from the University of Torino and the University of Hannover, carried on within the Personalized Information Systems working group of the REWERSE European Network of Excellence [8, 9, 11]. In this work, we faced the problem of curricula authoring and developed a layered architecture, inspired by [46, 45], that harmonizes different kinds of knowledge and of activity, expressed at various abstraction levels. As for all Semantic Web applications, each layer represents knowledge according to a well-defined semantics, thus enabling the application of automatic reasoning techniques as well as the integration with the representations belonging to other abstraction layers. The presented proposal is an evolution of earlier works [14, 9, 13], where we applied semantic annotations to learning objects, with the aim of building compositions of new learning objects, based on the user's learning goals and exploiting planning techniques. That proposal was based on a different approach that relied on the experience of the authors in the use of techniques for reasoning about actions and changes which, however, did not allow to tackle with curricula models and to the related verification tasks.

In the design of the layered architecture, great attention has been posed on the representation of courses and curricula, and on how to define curricula models. The chosen approach relies on the notion of competence, thus introducing an abstract perspective, in which courses do not directly depend on one another but rather have knowledge prerequisites and effects on the learner model $[10,27]$. Based on competences it is also possible to define pedagogical guidelines and rules that a curriculum must respect. To this aim, we have identified a set of inter-conceptual constraints and defined DCML, a graphical language for designing curricula models. As described in the previous sections, DCML allows the representation of temporal constraints posed on the acquisition of competences (supplied by courses), taking into account both the concepts supplied/required and the proficiency level. The language has a grounding in Linear Temporal Logic [28] and, hence, allows the application of various forms of reasoning and, in particular, to execute validation tasks like: the verification that a curriculum does not have competence gaps, the verification that a curriculum allows the acquisition of a learning goal, the verification that a curriculum satisfies the constraints contained in a curricula model.

We have shown how model checking techniques [23] can be used to execute all these validation tasks. This use of model checking is inspired by [60], where LTL formulas are used to describe and verify the properties of a composition of Web Services. Another recent work that inspired this proposal is [59], where medical guidelines, represented by means of the GLARE graphical language, are translated in a Promela program, whose properties are verified by using SPIN. Similarly to [59], the use of SPIN gives an automaton-based semantics to a curriculum - the automaton generated by SPIN from the Promela program -. The declarative, formal, representation of curricula models is instead given as a set of LTL constraints. This representation enables other forms of reasoning. In fact, as for all logical theories, we can use an inference engine to derive other theorems or to discover inconsistencies in the theory itself.

The underlieing assumption that we have done is that compentencies are given in terms of a shared vocabulary, which is known by all the involved actors, i.e learners, course creators, 
instructional designers. This is a common assumption in the Semantic Web field, and it is a necessary assumption for allowing the interoperability of the actors, mediated by the application [36]. This assumption, however, is very strong if we think to a realistic application. In this case, it is, in fact, quite likely that different actors and different schools use different vocabularies. This is a problem to face if we mean the proposed techniques to be used in the real world. We have already generally mentioned ontology alignment, merging and integration [30]. More practically, one possible, emerging solution for coping with this problem could be to rely on upper ontologies. Upper ontologies have, in fact, been proposed for being used as a lingua franca, to which mapping different vocabularies [43] in an automatic way. Specifically for what concerns the curriculum sequencing task, the implementation that we have described uses SWI-Prolog, which is thought for the integration with Semantic Web technology and provides a library for dealing with RDF. Even though in this work we have considered resources to be courses, the proposed representation is general and can, actually, be applied to any kind of learning resource. In the general case, the basic representation of resources, given in terms of competences, can also easily be enriched by adding media-related information and accessibility information, expressing constraints on how a resource is to be played/used. This information differs, in quality, from competence-based preconditions; in fact, while the latter only concerns the learner's knowledge, the former concerns some either environmental or physical aspect concerning the ability and the possibility of using the resource. So, for instance, a visually-impaired learner cannot watch an explanatory animation. For what concerns resources, this kind of information could be added to the precondition by enriching the annotation. On the other hand, the accessibility constraints of a learner could be added to the learner model. It is easy to extend the validation and the planning functionalities so to take into account also this kind of information.

In this article, we have also reported about the integration of the above approach into the Personal Reader Framework [38]. Despite some manual post-processing for fixing inconsistencies, we used real information from the Hannover University database of courses for extracting the meta-data. Currently the courses are annotated also by meta-data concerning the schedule, given in terms of semesters, and location of courses, like for instance room-numbers, addresses and teaching hours. This information is not used by the implemented services yet but it would be interesting to develop, as future work, also other services, which, along the lines of [64], complete the curricula planning process and produce an actual schedule.

The Personal Reader Platform provides a framework for implementing a service-oriented approach to personalization in the Semantic Web, and supplies a suitable infrastructure for building personalization applications, that consist of re-usable and interoperable personalization functionalities. The idea of taking a service-oriented approach to personalization is quite new and was born within the personalization working group of the Network of Excellence REWERSE. The adoption of a service-oriented architecture for the Personal Reader makes the introduction of new personalization functionalities very easy because each service focuses on dealing with a specific kind of knowledge and on performing a well-identified task, and is developed independently from the other services. Depending on the available resources and on the user's desires, it is possible to combine different services so to compose the functionalities that they implement, and, thus, perform more complex personalization tasks.

As future work, it will also be interesting to complement the architecture by integrating Web 2.0 features and develop personalization functionalities for handling information extracted from the activity of the community of users. This seems a very promising direction for recommendation purposes. For instance, when a user is uncertain on which curriculum to choose between two offers that are equivalent from the point of view of the supplied knowledge, he/she could be recommended to select one of the two on the basis of the behavior of other members in the community that we know as being "friends" of the current user.

\section{Acknowledgements}

The authors would like to thank prof. Giuseppe Berio for the helpful discussions. This research has partially been funded by the European Commission and by the Swiss Federal Office for Education 
and Science within the 6th Framework Programme project REWERSE number 506779 (cf. http: $/ /$ rewerse.net) and by DAAD and Ateneo Italo-Tedesco though the Vigoni German and Italian researchers exchange Program 2007-2008.

\section{References}

[1] IRS-III. Knowledge Media Institute, the Open University, Milton Keynes, UK, http://kmi . open.ac.uk/technologies/irs/. pages 18

[2] Unified Modeling Language: Superstructure, version 2.1.1. OMG, Object Management Group, February 2007. pages 5, 13

[3] F. Abel, I. Brunkhorst, N. Henze, D. Krause, K. Mushtaq, P. Nasirifar, and K. Tomaschweski. Personal reader agent: Personalized access to configurable web services. Technical report, Distributed Systems Institute, Semantic Web Group, University of Hannover, 2006. http: //www. personal-reader.de/wp/publications. pages 18

[4] G. Antoniou, E. Franconi, and F. van Harmelen. Introduction to semantic web ontology languages. In Eisinger and Maluszynski [27], pages 1-21. pages 4

[5] G. Antoniou, U. Uwe Aßmann, C. Baroglio, S. Decker, N. Henze, P.-L. Patranjan, and R. Tolksdorf, editors. Reasoning Web, Third International Summer School 2007, Dresden, Germany, September 3-7, 2007, Tutorial Lectures, volume 4636 of Lecture Notes in Computer Science. Springer, 2007. pages 2

[6] G. Antoniou and F. van Harmelen. A Semantic Web Primer. the MIT Press, 2004. pages 2

[7] M. Baldoni, C. Baroglio, I. Brunkhorst, N. Henze, E. Marengo, and V. Patti. A Personalization Service for Curriculum Planning. In E. Herder and D. Heckmann, editors, Proc. of the 14 th Workshop on Adaptivity and User Modeling in Interactive Systems, ABIS 2006, pages 17-20, Hildesheim, Germany, October 2006. pages 21

[8] M. Baldoni, C. Baroglio, I. Brunkhorst, E. Marengo, and V. Patti. Reasoning-based Curriculum Sequencing and Validation: Integration in a Service-Oriented Architecture. In E. Duval and R. Klamma, editors, Proc. of EC-TEL 2007 - 2nd Europ. Conf. on Technology Enhanced Learning, volume 4756 of LNCS, pages 426-431. Springer, 2007. pages 30

[9] M. Baldoni, C. Baroglio, and N. Henze. Personalization for the Semantic Web. In N. Eisinger and J. Maluszynski, editors, Reasoning Web, First International REWERSE Summer School 2005, volume 3564 of LNCS Tutorials, pages 173-212. Springer-Verlag, Malta, July 2005. pages $5,13,30$

[10] M. Baldoni, C. Baroglio, N. Henze, and V. Patti. Setting up a framework for comparing adaptive educational hypermedia: First steps and application on curriculum sequencing. In N. Henze, editor, Proc. of ABIS-Workshop 2002: Personalization for the mobile World, Workshop on Adaptivity and User Modeling in Interative Software Systems, pages 43-50, Hannover, Germany, October 2002. pages 5, 30

[11] M. Baldoni, C. Baroglio, and E. Marengo. Curricula modeling and checking. In Proc. of the 10th Congress of the Italian Association for Artificial Intelligence, AI*IA 2007, volume 4733 of $L N C S$, pages 471-482, 2007. pages 14, 30

[12] M. Baldoni, C. Baroglio, A. Martelli, V. Patti, and L. Torasso. Verifying the compliance of personalized curricula to curricula models in the semantic web. In M. Bouzid and N. Henze, editors, Proc. of the Semantic Web Personalization Workshop, held in conjuction with the 3rd European Semantic Web Conference, pages 53-62, Budva, Montenegro, 2006. pages 5, 13 
[13] M. Baldoni, C. Baroglio, and V. Patti. Web-based adaptive tutoring: an approach based on logic agents and reasoning about actions. Artificial Intelligence Review, 22(1):3-39, 2004. pages $1,5,7,13,22,30$

[14] M. Baldoni, C. Baroglio, V. Patti, and L. Torasso. Reasoning about learning object metadata for adapting SCORM courseware. In L. Aroyo and C. Tasso, editors, AH 2004: Workshop Proceedings, Part I, International Workshop on Engineering the Adaptive Web, EAW'04: Methods and Technologies for personalization and Adaptation in the Semantic Web, pages 4-13, Eindhoven, The Netherlands, August 2004. Technische Universiteit Eindhoven. pages 5,30

[15] R. Baumgartner, S. Flesca, and G. Gottlob. Visual web information extraction with lixto. In Peter M. G. Apers, Paolo Atzeni, Stefano Ceri, Stefano Paraboschi, Kotagiri Ramamohanarao, and Richard T. Snodgrass, editors, VLDB, pages 119-128. Morgan Kaufmann, 2001. pages 19

[16] R. Baumgartner, N. Henze, and M. Herzog. The personal publication reader: Illustrating web data extraction, personalization and reasoning for the semantic web. In $E S W C$, pages 515-530, 2005. pages 3, 18, 19

[17] Tim Berners-Lee. Semantic web - keynote at xml 2000 conference, 2000. http://www.w3.org/2000/Talks/1206-xml2k-tbl/slide10-0.html. pages 1

[18] Tim Berners-Lee. The semantic web - mit/lcs seminar, 2002. http://www.w3c.org/2002/Talks/09-lcs-sweb-tbl/. pages 1

[19] Aoife Brady, Owen Conlan, Vincent Wade, and Declan Dagger. Supporting Users in Creating Pedagogically Sound Personalised Learning Objects. In Adaptive Hypermedia and Adaptive Web-Based Systems, pages 52-61, 2008. pages 3

[20] P. Brusilovsky. Course sequencing for static courses? applying ITS techniques in large-scale web-based education. Intelligent tutoring systems, pages 625-634, 2000. pages 28

[21] P. Brusilovsky and J. Vassileva. Course sequencing techniques for large-scale web-based education. Int. J. Cont. Engineering Education and Lifelong learning, 13(1/2):75-94, 2003. pages $1,6,7,27,28,29$

[22] C. Castro and S. Manzano. Variable and value ordering when solving balanced academic curriculum problems. In Proc. of the 6th Workshop of ERCIM WG on Constraints, 2001. pages 29

[23] O. E. M. Clarke and D. Peled. Model checking. MIT Press, Cambridge, MA, USA, 2001. pages 6,30

[24] Learning Technology Standards Committee. Ieee standard for learning object metadata, 2002. IEEE Standard 1484.12.1, Institute of Electrical and Electronics Engineers. pages 2

[25] Owen Conlan. Towards a Standards-based Approach to e-Learning Personalization using Reusable Learning Objects. In Proc. of World Conference on E-Learning, E-Learn 2002, pages 210-217, 2002. pages 1, 2

[26] J. L. De Coi, E. Herder, A. Koesling, C. Lofi, D. Olmedilla, O. Papapetrou, and W. Sibershi. A model for competence gap analysis. In WEBIST 200\%, Proceedings of the Third International Conference on Web Information Systems and Technologies: Internet Technology / Web Interface and Applications, Barcelona, Spain, Mar 2007. INSTICC Press. pages 4, 6

[27] Norbert Eisinger and Jan Maluszynski, editors. Reasoning Web, First International Summer School 2005, Msida, Malta, July 25-29, 2005, Tutorial Lectures, volume 3564 of Lecture Notes in Computer Science. Springer, 2005. pages 30, 32 
[28] E. A. Emerson. Temporal and modal logic. In Handbook of Theoretical Computer Science, volume B, pages 997-1072. Elsevier, 1990. pages 2, 5, 7, 30

[29] European Commission, Education and Training. The Bologna process. http://europa.eu.int/comm/education/policies/educ/bologna/bologna_en.html. pages 2

[30] Jérôme Euzenat and Pavel Shvaiko. Ontology matching. Springer-Verlag, Heidelberg (DE), 2007. pages 5,31

[31] R. Farrell, S. D. Liburd, and J. C. Thomas. Dynamic assembly of learning objects. In Proc. of $W W W 2004,2004$. pages 29

[32] M. del Mar Gallardo, P. Merino, and E. Pimentel. Debugging UML Designs with Model Checking. Journal of Object Technology, 1(2):101-117, July-August 2002. pages 15

[33] A. Garro, N. Leone, and F. Ricca. Logic Based Agents for E-learning. In Proc. of the IJCAI'03 Workshop on Knowledge Representation and Automated Reasoning for E-Learning Systems, pages $36-45,2003$. pages 28

[34] A. Garro, L. Palopoli, and F. Ricca. Exploiting agents in e-learning and skills management context. AI Commun., 19(2):137-154, 2006. pages 28

[35] N. Guelfi and A. Mammar. A Formal Semantics of Timed Activity Diagrams and its PROMELA Translation. In Proc. of the 12th Asia-Pacific Software Engineering Conference (APSEC'05), pages 283-290. IEEE Computer Society, 2005. pages 15

[36] Richard Hackelbusch and Hans-Jürgen Appelrath. A Distributed Ontological Approach as a Basis for Software in the Context of Academic Programs. In EC-TEL, pages 122-127, 2008. pages $5,30,31$

[37] N. Henze. Personal readers: Personalized learning object readers for the semantic web. In 12th International Conference on Artificial Intelligence in Education, AIED05, Amsterdam, The Netherlands, 2005. pages 18

[38] N. Henze and D. Krause. Personalized access to web services in the semantic web. In The 3rd International Semantic Web User Interaction Workshop (SWUI, collocated with ISWC 2006, November 2006. pages 3, 7, 18, 19, 21, 31

[39] N. Henze and W. Nejdl. Adaptation in open corpus hypermedia. IJAIED Special Issue on Adaptive and Intelligent Web-Based Systems, 12:325-350, 2001. pages 1, 7

[40] G. J. Holzmann. The SPIN Model Checker, Primer and Reference Manual. Addison-Wesley, 2003. pages $6,7,15$

[41] T. Lambert, C. Castro, E. Monfroy, and F. Saubion. Solving the balanced academic curriculum problem with an hybridization of genetic algorithm and constraint propagation. In L. Rutkowski, R. Tadeusiewicz, L. A. Zadeh, and J. Zurada, editors, Artificial Intelligence and Soft Computing, ICAISC 2006, volume 4029 of LNCS, pages 410-419. Springer, 2006. pages 29

[42] Jennifer Lennon and Hermann Maurer. Why it is Difficult to Introduce e-Learning into Schools And Some New Solutions. in journal J.UCS, 9:1244-1257, 2003. pages 1, 2

[43] V. Mascardi, P. Rosso, and V. Cord. Enhancing Communication inside Multi-Agent Systems. An Approach based on Allignment via Upper Ontologies. In M. Baldoni, C. Baroglio, and V. Mascardi, editors, Proceedings of MALLOW-AWESOME'00\%. First Workshop on Agents, Web-Services, and Ontologies., pages 92-107, 2007. pages 31 
[44] M. Melia and C. Pahl. Automatic Validation of Learning Object Compositions. In Information Technology and Telecommunications Conference ITET'2005: Doctoral Symposium, Carlow, Ireland, 2006. pages 27

[45] Mark Melia and Claus Pahl. An information architecture for validating courseware. In LODE, 2007. pages $2,3,5,6,27,30$

[46] Mark Melia and Claus Pahl. Pedagogical validation of courseware. In EC-TEL, pages 499-504, 2007. pages $2,3,5,6,14,27,30$

[47] P. Mohan and C. Brooks. Learning Object on the Semantic Web. In Proc. of the 3rd IEEE International Conference onAdvanced Learning Technologies, Athens, Greece, 2003. pages 2

[48] A. Paschke and Y. Biletskiy, editors. Advances in Rule Interchange and Applications. Number 4824 in LNCS. Springer, 2007. pages 2

[49] L. Pazzi. Three points of view in the characterization of complex entities. In N. Guarino, editor, Formal Ontology in Information Systems. IOS Press, 1998. pages 4

[50] D. Persico. Courseware validation: a case study. Journal of Computer Assisted Learning, 12:232-244, 1996. pages 7

[51] O. Pistamiglio. Sviluppo di un plugin grafico per eclipse: DCML Designer. Laurea specialistica in informatica, Corso di Studi in Informatica, Università degli Studi di Torino, 2007. Supervisor: Prof. M. Baldoni. pages 23

[52] REWERSE. Reasoning on the Web with Rules and Semantics. http://www.rewerse.net. pages 2

[53] D. Roman, U. Keller, H. Lausen, J. de Bruijn, R. Lara, M. Stollberg, Axel Polleres, C. Feier, C. Bussler, and D. Fensel. Web service modeling ontology. Applied Ontology, 1(1):77-106, 2005. pages 18

[54] P.V. Rosmalen, H. Vogten, R.V. Es, H. Passier, P. Poelmans, and R. Koper. Authoring a full life cycle model in standards-based, adaptive e-learning. Journal of Educational Technology and Society, 9(1):72-83, 2006. pages 7

[55] Stuart Russell and Peter Norvig. Artificial Intelligence, A Modern Approach, Second Edition. Prentice Hall Series in Artificial Intelligence, 2003. pages 7

[56] J.W. Samples. The pedagogy of technology - our next frontier? Connexions, 14(2):4-5, 2002. pages 2

[57] S. Schaffert, F. Bry, S. Berger, T. Furche, P. Patranjan, and M. Eckert. Xcerpt - rule-based querying and reasoning on the (semantic) web. http://www.xcerpt.org/documentation/spec/. pages 2

[58] L. Stojanovic, S. Staab, and R. Studer. eLearning based on the Semantic Web. In WebNet2001 - World Conference on the $W W W$ and Internet, Orlando, Florida, USA, 2001. pages 2

[59] P. Terenziani, L. Giordano, A. Bottrighi, S. Montani, and L. Donzella. SPIN Model Checking for the Verification of Clinical Guidelines. In Proc. of ECAI 2006 Workshop on AI techniques in healthcare: evidence-based guidelines and protocols, Riva del Garda, August 2006. pages 30

[60] W. M. P. van der Aalst and M. Pesic. DecSerFlow: Towards a Truly Declarative Service Flow Language. In Mario Bravetti and Gialuigi Zavattaro, editors, Proc. of WS-FM, LNCS, Vienna, September 2006. Springer. pages 5, 30 
[61] J. Vassileva. Dynamic CAL-Courseware Generation Within an ITS-Shell Architecture. In I. Tomek, editor, Computer Assisted Learning, volume 601 of LNCS, pages 581-591. Springer, 1992. pages 1,7

[62] W3C. OWL Web Ontology Language Semantics and Abstract Syntax. Available at http://www.w3.org/TR/owl-semantics/. pages 2

[63] W3C. RDF Primer. Available at http://www.w3.org/TR/rdf-primer/. pages 2

[64] K. Wu and W. S. Havens. Modelling an academic curriculum plan as a mixed-initiative constraint satisfaction problem. In B. Kégl and G. Lapalme, editors, Canadian Conference on AI, volume 3501 of $L N C S$, pages 79-90, 2005. pages 12, 29, 31 